Proc. Estonian Acad. Sci. Biol. Ecol., 2005, 54, 2, 136-165

\title{
Annotated checklist and distribution of the true bugs (Hemiptera-Heteroptera) of Estonia
}

\author{
Carl-Cedric Coulianos \\ Kummelnäsvägen 90, S-132 37 Saltsjö-Boo, Sweden; cc.coulianos@zeta.telenordia.se \\ Received 14 November 2003, in revised form 31 May 2004
}

\begin{abstract}
On the basis of recent fieldwork, studies of museum and private collections and faunistic literature 454 species of true bugs are listed from Estonia. Their distribution is presented by their occurrence in the 14 UTM grid $100 \times 100 \mathrm{~km}$ squares covering Estonia. Faunistic remarks to 58 species are added. A list with comments on species erroneously published from Estonia is given. The following species are reported as new to Estonia: Plea minutissima (Leach), Dicyphus epilobii Reuter, and Globiceps fulvicollis Jakovlev.
\end{abstract}

Key words: Hemiptera, Heteroptera, checklist, Estonian fauna.

\section{INTRODUCTION}

The investigation of the true bugs of Estonia started in the 1850s when Gustav Flor (1829-1883), a medical doctor and professor in Dorpat (= Tartu), began to collect and study the Hemiptera in Livonia (= present South Estonia and North Latvia). His collecting localities are listed with present-day names by Vilbaste (1973b) and Lukashuk (1997). Flor's studies resulted in his now classic, twovolume work Die Rhynchoten Livlands (Flor 1860, 1861), notable for his good descriptions. In these volumes he described 26 new species and one variety of Heteroptera, 12 of which are still valid. His collection is kept separately in the Institute of Zoology and Botany (IZB), Tartu, and it is in a very good condition.

After Flor's time nothing was published on Estonian Heteroptera until the 1920s when several articles appeared in different journals (Mühlen \& Schneider 1920, Bianchi \& Kiritshenko 1923, Stichel 1927). During the 1930s H. Haberman collected also Heteroptera, now in coll. IZB (Haberman 1933-1937) and several notes were published, especially on aquatic species (Mikkelsaar 1934, Sepp 1939) and also on a selection of species from Saaremaa (Kauri 1934) and Läänemaa (Haberman 1938). A very successful collector during that time was L. Voore (material in coll. IZB). She also published an interesting but much overlooked paper on the Estonian shore-bugs (Voore 1940) and compiled an unpublished list of Estonian Heteroptera, which is now in Estonian Naturalists' Society (ENS). 
During the 1940s and 1950s investigations were made on the fauna of Estonian bogs and marshes resulting in papers dealing also with Heteroptera by Jüris (1940), Maavara (1957), and Vilbaste $(1955,1958)$. Several investigations during the 1950s-1970s on the fauna of the river system of the Emajõgi River were summarized by Ristkok (1994).

Investigations of the fauna including Heteroptera from certain parts of Estonia have been published by Vilbaste (1970, 1973a, 1979, 1985), Mäemets (1975), Rebassoo (1987), and Luig \& Talvi (1993). Records of aquatic and semiaquatic Heteroptera have been published by H. Timm, T. Timm, and V. Timm (see References), Valk (1984), and Järvekülg (2001). Coulianos (1999, 2003) added 13 and 57 species, respectiveley, to the Estonian list. Records of some plant pest species have been reported by Leius (1940) (Miridae, Rhopalidae), Rõigas (1975), and Voolma (1992) (Aradus cinnamomeus).

During the last two decades much material of Heteroptera has been collected by K. Elberg, M. Kruus, M. Marits, M. Martin, A. Selin, H. Silfverberg, and the present author, all of which has been studied for the present checklist.

The following list shows the number of species of Heteroptera reported for Estonia in some published and unpublished works:

$\begin{array}{ll}\text { Flor }(1860,1861) & 210 \\ \text { Voore (1940, unpublished list in ENS) } & 307 \\ \text { Remm (1966) } & 335 \\ \text { Remm \& Viidalepp (1986, unpublished list in IZB) } & 357 \\ \text { Vilbaste \& Viidalepp (1988, unpublished list in IZB) } & 365 \\ \text { Lukashuk (1997) } & 275 \\ \text { The present checklist } & 454\end{array}$

The numbers are corrected with regard to synonyms, wrong determination, and misinterpreted localities. The numbers from Lukashuk (1997) are not comparable with the others as he mainly used published records but omitted Remm (1966) and overlooked some later papers.

\section{FORMAT OF THE CHECKLIST}

The checklist (Table 1) includes species with verified records in the collections studied by the present author. For five species only published, but reliable, records were available. The nomenclature within the families Ceratocombidae and Pyrrhocoridae is according to Aukema \& Rieger (1998-2001) and within the remaining families according to Lukashuk (1997). Within genera the species are arranged in alphabetical order. Synonyms are given only for species group names differing from the checklist in Remm (1966). All species are sequentially numbered throughout. An asterisk $(*)$ before the species number refers to the corresponding number in the Remarks section. The distribution of the species in Estonia is presentated as their occurrence in the 14 UTM grid system $100 \times 100 \mathrm{~km}$ squares that covers Estonia (Fig. 1).

In the columns for the different squares the following symbols are used: + records verified by the author; ? published, unverified records; - no records. 


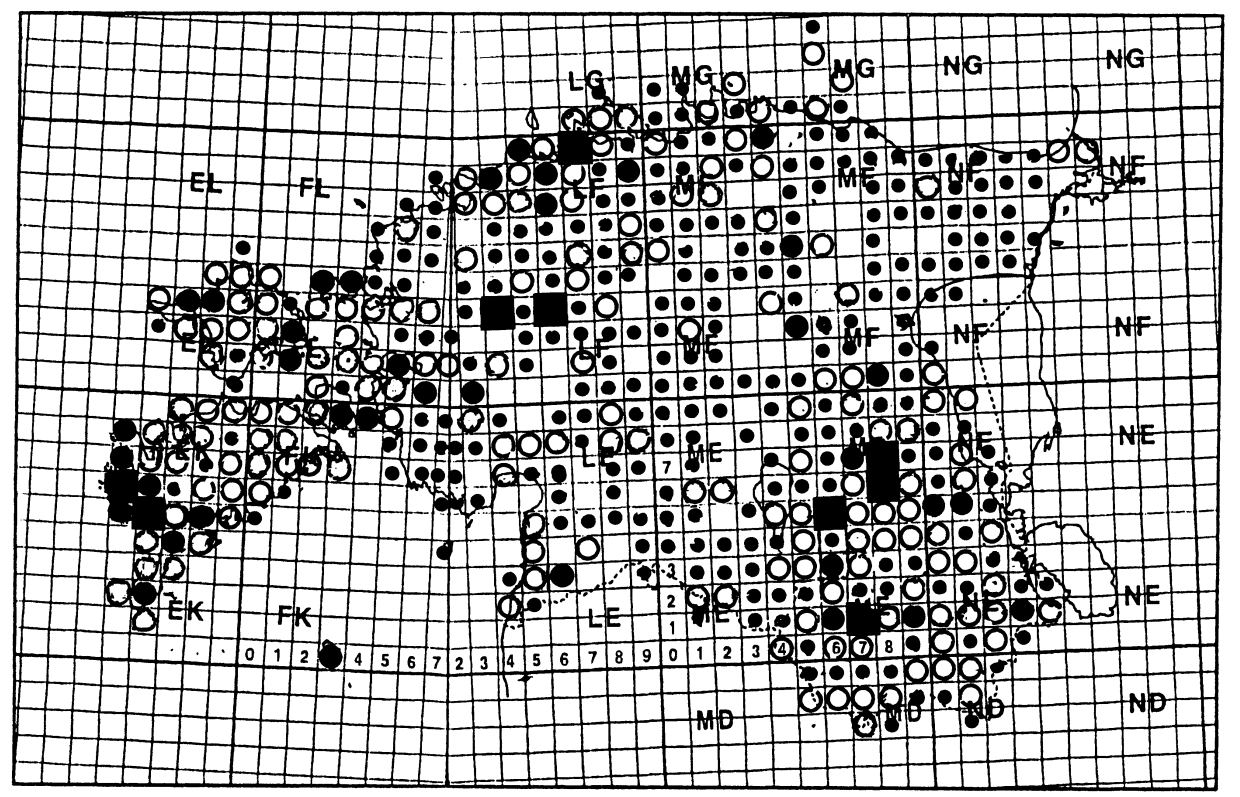

Fig. 1. Contour map of Estonia with the UTM grid system $(10 \times 10 \mathrm{~km})$. The capital letters denote the $100 \times 100 \mathrm{~km}$ squares used to present the distribution of the species in the checklist. The number of species recorded from each $10 \times 10 \mathrm{~km}$ square is shown by the following symbols: -1-10 spp., ○ 11-50 spp., ○ 51-99 spp., — 100- spp.

\section{STUDIED MATERIAL}

The following collections have been studied (with abbreviations used in the text): Institute of Zoology and Botany, Tartu (IZB); coll. Flor in IZB; Zoological Museum, University of Tartu (ZMT); Institute of Plant Protection, Estonian Agricultural University, Tartu (IPP); Estonian Museum of Natural History, Tallinn $(E M N)$; the private collections of Carl-Cedric Coulianos (Saltsjö-Boo, Sweden), Kaupo Elberg (Tartu), Märt Kruus (Tartu), Mati Marits (Tartu), Mati Martin (Tartu), Allan Selin (Tallinn), Hans Silfverberg (Helsinki), and Heino Õunap (Tartu).

The studied literature with faunistic data on Estonian Heteroptera is listed in References. A total of 26440 specimens have been determined and 16442 records were used by the author for the present checklist.

\section{SPECIES ERRONEOUSLY REPORTED FROM ESTONIA}

Among the species recorded from Estonia by Kiritshenko (1951) 25 are due to misinterpretation of localities in Flor $(1860,1861)$ actually in Latvia. Remm (1966) included 23 of these species in his checklist and added another 13 of 
which there are no published records or collection specimens collected before 1966. Of these 38 species, 1 has since been recorded by Lukashuk (1997) and 11 by Coulianos (2003). For the remaining 26 species listed below no verified records exist and they have to be excluded from the Estonian list.

Velia caprai Tamanini, 1947. Wrongly determined $=V$. saulii sec ex. in IZB.

Corixa punctata (Illiger, 1807). Wrongly determined $=C$. dentipes sec ex. in IZB.

Stethoconus cyrtopeltis (Flor, 1860). Misinterpretation of data for Latvia in Flor (1860).

Phytocoris varipes Boheman, 1852. Misinterpretation of data for Latvia in Flor (1860) (as P. ulmi).

Note: all specimens under the name P. ulmi in coll. Flor are P. insignis Reuter, 1876.

Atractotomus kolenatii (Flor, 1860). Misinterpretation of data for Latvia in Flor (1860).

Parapsallus vitellinus (Scholtz, 1847). Misinterpretation of data for Latvia in Flor (1860).

Psallus wagneri Ossiannilsson, 1953. Wrongly determined $=P$. perrisi sec ex. in IZB.

Sthenarus rotermundi (Scholtz, 1847). Misinterpretation of data for Latvia in Flor (1860).

Agramma confusum (Puton, 1879). Recorded as v. fallax Horváth, 1906 by Stichel (1927). Doubtful record, refers probably to $A$. femorale.

Agramma laetum (Fallén, 1807). Wrongly determined = A. femorale sec ex. in IZB.

Lasiacantha capucina (Germar, 1837). Misinterpretation of data for Latvia in Flor (1860).

Acompocoris alpinus Reuter, 1875. Based on unverified supposal that lucorum sensu Flor partly belongs to this species. See Lukashuk (1997).

Aradus brevicollis Fallén, 1807. Misinterpretation of data for Latvia in Flor (1860).

Aradus pictus auct. non Baerensprung, 1859 = obtectus Vasarhelyi, 1988. Misinterpretation of data for Latvia in Flor (1860).

Mezira tremulae (Germar, 1822). Misinterpretation of data for Latvia in Flor (1860).

Tropidothorax leucopterus (Goeze, 1778). An error by Kiritshenko (1951) due to an unlabelled specimen in coll. Gimmerthal mentioned by Flor (1860).

Enoplops scapha (Fabricius, 1794). Doubtful record depending on unlabelled specimen in coll. Gimmerthal mentioned by Flor (1860).

Cydnus aterrimus (Forster, 1771). Included by Remm (1966) on the basis of a specimen from the 19th century, 1 q labelled "Est.?" in coll. IZB. A doubtful record.

Microporus nigritus (Fabricius, 1794). An error by Kiritshenko (1951), Remm (1966).

Canthophorus dubius (Scopoli, 1763). Misinterpretation of data for Latvia in Flor (1860). Note: the specimens in coll. Flor from Latvia belong to C. impressus Horváth, 1880.

Sehirus morio (Linnaeus, 1761). Wrongly determined = S. luctuosus sec ex. in IZB.

Podops inunctus (Fabricius, 1775). Misinterpretation of data for Latvia in Flor (1860).

Carpocoris pudicus (Poda, 1761). Wrongly determined $=$ C. purpureipennis sec ex. in IZB.

Holcostethus sphacelatus (Fabricius, 1794). Misinterpretation of data for Latvia in Flor (1860), which, however, is a doubtful record from unlabelled specimen in coll. Gimmerthal.

Eurydema ornatum (Linnaeus, 1758). Wrongly determined $=$ E. dominulus. All specimens from Estonia seen by me belong to the latter species.

Arma custos (Fabricius, 1794). Recorded by Remm (1966) based on three specimens from Koshelki, Vasina-Gora 31.v.1938 leg. L. Voore, coll. IZB. This locality is now in Russia.

\section{GENERAL REMARKS}

True bugs have been recorded from most parts of Estonia. There are records from $465(82 \%)$ of the $56710 \times 10 \mathrm{~km}$ UTM squares falling fully or partially within the boundaries of the Estonian territory (Fig. 1). However, it is evident that 
the investigation effort has been different for the various regions. This is reflected in the number of species recorded from each $10 \times 10 \mathrm{~km}$ square as well as the total number of species found in the $14100 \times 100 \mathrm{~km}$ squares. From only nine $10 \times 10 \mathrm{~km}$ squares 100 species or more have been recorded, with a maximum of 194 species in ME 87.

This is also shown by Fig. 2 where the combined records of five very common, abundant and widely distributed species are mapped. These species represent four major habitats (aquatic, ground, field layer, trees and bushes). At least one of these species can be expected to occur in every $10 \times 10 \mathrm{~km}$ square in Estonia. The existence of under-collected parts of the country is quite evident. The best known parts of Estonia are in the west (including the western islands), north (the Tallinn area), and south-east (the Tartu area).

As is evident from the Introduction, the investigation effort has been different during various periods of time. The following 12 species have only been recorded before 1900: Saldula melanoscela, Dicyphus stachydis, Deraeocoris punctulatus, Globiceps sphegiformis, Orthotylus virens, Conostethus roseus, Derephysia cristata, Xylocoris galactinus, Aradus aterrimus, Heterogaster urticae, Raglius alboacuminatus, and Spathocera dalmannii. Another 52 species have only been recorded during the period 1900-1979. During the period 1980-2003 there are records of 390 species, i.e. $86 \%$ of the species known from Estonia at present. The total

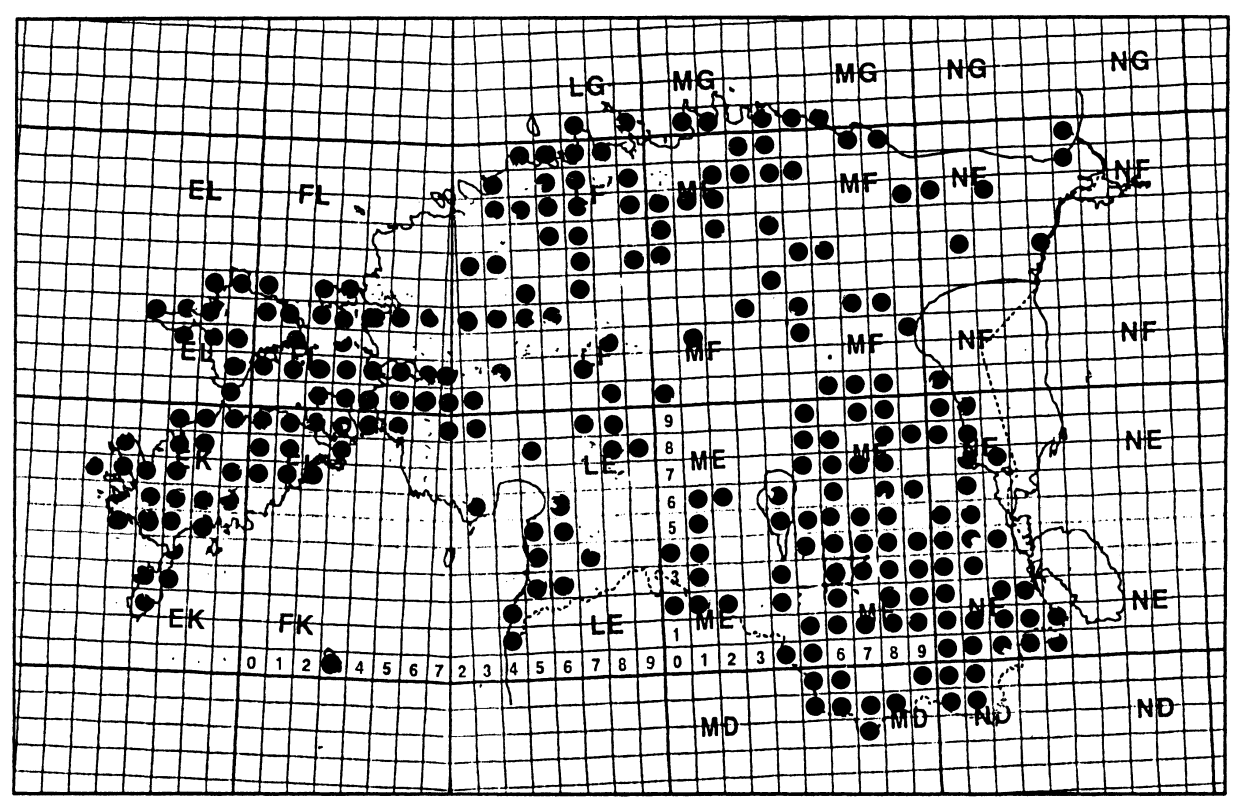

Fig. 2. Map of the $10 \times 10 \mathrm{~km}$ UTM squares with records of one or more of the following common and widely distributed species Gerris lacustris, Lygus rugulipennis, Anthocoris nemorum, Nysius thymi, and Dolycoris baccarum. 
number of species (454) recorded from Estonia is high in comparison with the other Baltic countries, which, however, are not sufficiently investigated. The following numbers of species are known from the neighbouring countries (with the percentage of the Estonian species known from each country): Lithuania 281 (52.2\%), Latvia 384 (76.9\%), Finland 486 (88.3\%), Sweden 601 (94.3\%). Not known from either Latvia or Lithuania are 89 species and not known from either Finland or Sweden are 14 Estonian species.

The following species recorded from Estonia are not known from any of the four countries above: Agnocoris reclairei, Capsus pilifer, Globiceps sphegiformis, Heterocordylus erythrophthalmus, Sciocoris macrocephalus, and Antheminia aliena. (See Remarks to these species.)

The fauna of true bugs of Latvia and Lithuania is still too unsatisfactorily known to permit a discussion of the Estonian fauna in a wider Baltic context. However, based on the fauna of the neighbouring countries, it can be estimated that $90-95 \%$ of the species of true bugs actually occurring in Estonia have been recorded here.

Table 1. Checklist and distribution of the true bugs (Hemiptera-Heteroptera) of Estonia by UTM grid $100 \times 100 \mathrm{~km}$ squares

\begin{tabular}{l|l|l|l|l|l|l|l|l|l|l|l|l|l|l} 
No. & LG & MG & EL & FL & LF & MF & NF & EK & FK & LE & ME & NE & MD & ND \\
\hline
\end{tabular}

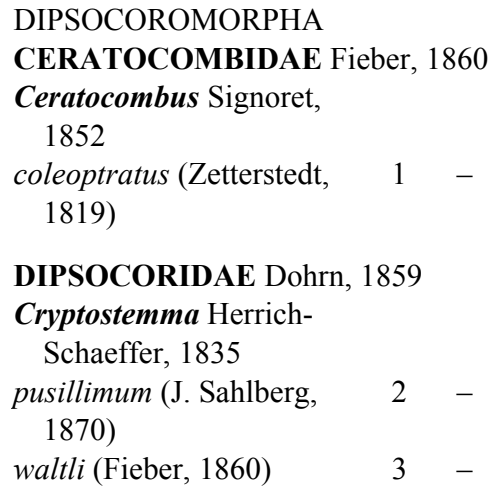

GERROMORPHA

MESOVELIIDAE Douglas \& Scott, 1852

Mesovelia Mulsant \& Rey,

1852

furcata Mulsant \& Rey, 4 1852

HEBRIDAE Amyot \& Serville, 1843

Hebrus Curtis, 1833 pusillus (Fallén, 1807)

ruficeps Thomson, 1871

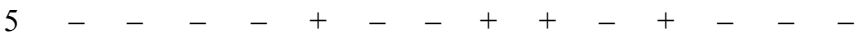

$$
\begin{aligned}
& 6-+\ldots++\quad+\quad+\quad+\quad+\quad+
\end{aligned}
$$

Continued overleaf 
Table 1. Continued

\begin{tabular}{l|l|l|l|l|l|l|l|l|l|l|l|l|l|l} 
No. & LG & MG & EL & FL & LF & MF & NF & EK & FK & LE & ME & NE & MD & ND \\
\hline
\end{tabular}

HYDROMETRIDAE Billberg, 1820

Hydrometra Latreille, 1796

gracilenta Horváth, 1899

VELIIDAE Brullé, 1836

Microvelia Westwood,

1834

buenoi Drake, 1920

= umbricola

Wroblewski, 1938

reticulata (Burmeister,

Velia Latreille, 1804

saulii Tamanini, 1947

10

GERRIDAE Leach, 1815

Aquarius Schellenberg,

1800

najas (DeGeer, 1773)

paludum (Fabricius, 1794)

Gerris Fabricius, 1794

argentatus Schummel, $183213-{ }_{-}+{ }_{+}++++++\quad+\quad+\quad+$

lacustris (Linnaeus, 1758) $14++++++++++++++$

lateralis Schummel, $1832 \quad 15-{ }_{-}+{ }_{-}+{ }_{+}+{ }_{+}++_{+}+{ }_{+}+$

odontogaster (Zetterstedt, $16+++++++++++++++$

1828)

thoracicus Schummel, 1832

Limnoporus Stål, 1868

rufoscutellatus (Latreille,

1807)

NEPOMORPHA

NEPIDAE Latreille, 1802

Nepa Linnaeus, 1758

cinerea Linnaeus, 1758

Ranatra Fabricius, 1790

linearis (Linnaeus, 1758)

CORIXIDAE Leach, 1815

Micronecta Kirkaldy, 1897

griseola Horváth, 1899

minutissima (Linnaeus,

$* 21$

1758)

poweri (Douglas \& Scott,

1869)

Cymatia Flor, 1860

bonsdorffii (C. R.

Sahlberg, 1819) 
Table 1. Continued

\begin{tabular}{|c|c|c|c|c|c|c|c|c|c|c|c|c|c|c|}
\hline & No. & LG & $\mathrm{MG}$ & EL & FL & LF & MF & $\mathrm{NF}$ & EK & FK & LE & $\mathrm{ME}$ & $\mathrm{NE}$ & $\mathrm{MD}$ \\
\hline
\end{tabular}
1777)

Glaenocorisa Thomson,

1869

cavifrons (Thomson, 1869) *26

propinqua (Fieber, 1860) *27

Callicorixa White, 1873

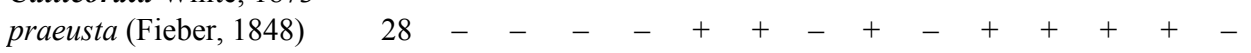

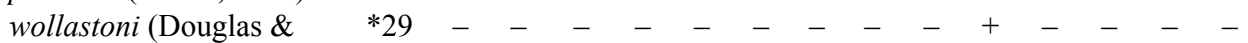

Scott, 1865)

Corixa Geoffroy, 1762

dentipes Thomson, 1869

Hesperocorixa Kirkaldy, 1908

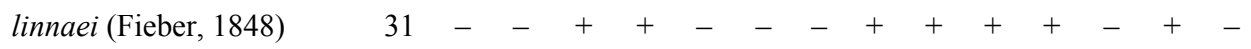

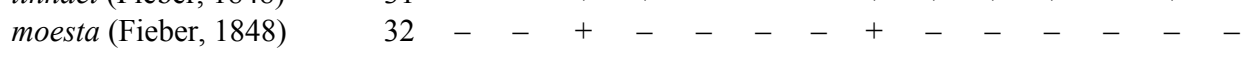

sahlbergi(Fieber, 1848) $33-{ }_{-}+{ }_{+}+{ }_{+}+{ }_{+}+{ }_{+}++_{+}+$

Paracorixa Poisson, 1957

concinna (Fieber, 1848)

Sigara Fabricius, 1775

distincta (Fieber, 1848) $35+-{ }_{-}+{ }_{+}+-{ }_{+}+{ }_{+}+{ }_{+}+-$

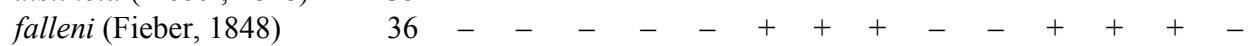

fossarum (Leach, 1817) $37-c_{-}+{ }_{-}+{ }_{-}+{ }_{-}+{ }_{-}-{ }_{-}-$

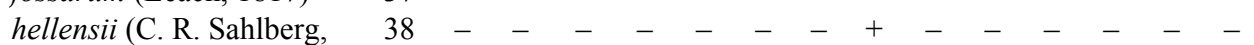

1819)

lateralis (Leach, 1817)

longipalis (J. Sahlberg,

1878)

nigrolineata (Fieber, 1848)

scotti (Douglas \& Scott,

1868)

semistriata (Fieber, 1848)

striata (Linnaeus, 1758)

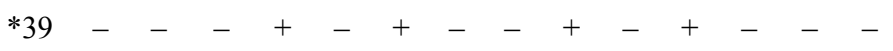

*40 - $\quad-\quad-\quad-\quad+\quad+\quad-\quad-\quad-\quad-\quad-\quad+-$

$41-\quad-\quad-\quad-\quad+\quad-\quad-\quad+\quad-\quad+\quad+\quad-\quad-$

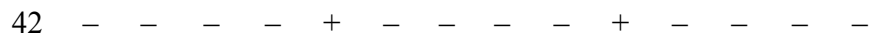

$\begin{array}{llllllllllllllll}43 & + & + & + & + & + & + & + & + & + & + & + & + & - & - \\ 44 & + & + & - & + & + & + & - & + & + & + & + & + & + & -\end{array}$

NAUCORIDAE Leach, 1815

Ilyocoris Stål, 1861

cimicoides (Linnaeus, 1758)

APHELOCHEIRIDAE Fieber, 1851

Aphelocheirus Westwood,

1833

aestivalis (Fabricius, 1794) 46

NOTONECTIDAE Latreille, 1802

Notonecta Linnaeus, 1758

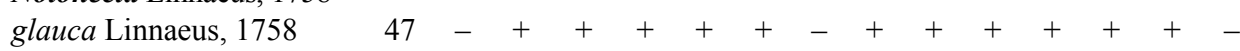

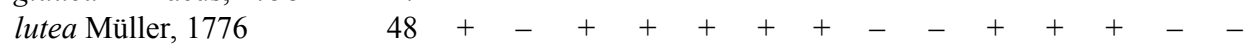

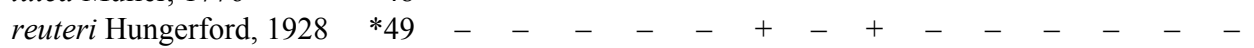

Continued overleaf 
Table 1. Continued

\begin{tabular}{l|l|l|l|l|l|l|l|l|l|l|l|l|l|l} 
No. & LG & MG & EL & FL & LF & MF & NF & EK & FK & LE & ME & NE & MD & ND \\
\hline
\end{tabular}

PLEIDAE Fieber, 1851

Plea Leach, 1817

minutissima Leach, 1817

LEPTOPODOMORPHA

SALDIDAE Amyot \& Serville, 1843

Chartoscirta Stål, 1868

cincta (Herrich-Schaeffer, 51

1841)

cocksii (Curtis, 1835)

elegantula (Fallén, 1807)

Halosalda Reuter, 1912

lateralis (Fallén, 1807)

Macrosaldula Leston \&

Southwood, 1964

scotica (Curtis, 1835)

Micracanthia Reuter, 1912

fennica (Reuter, 1884)

marginalis (Fallén, 1807)

Saldula VanDuzee, 1914

arenicola (Scholtz, 1847)

c-album (Fieber, 1859)

fucicola (J. Sahlberg, 1870) *60

melanoscela (Fieber, 1859) 61

opacula (Zetterstedt, 1838)

orthochila (Fieber, 1859)

pallipes (Fabricius, 1794)

palustris (Douglas, 1874)

pilosella (Thomson, 1871)

saltatoria (Linnaeus, 1758)

Salda Fabricius, 1803

littoralis (Linnaeus, 1758) 68

morio Zetterstedt, 1838

muelleri (Gmelin, 1790) *70

sahlbergi Reuter, 1875

$\begin{array}{llllllllllllllll}52 & - & - & - & - & - & - & - & + & - & - & - & - & - & - \\ 53 & - & - & - & - & - & - & + & + & + & - & + & + & - & +\end{array}$

$54-\quad+\quad+\quad+\quad-\quad-\quad-\quad+\quad+\quad-\quad-\quad-\quad-\quad-$

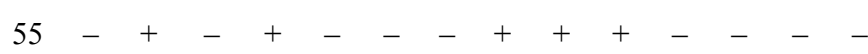

$56-\quad-\quad+\quad+\quad+-\quad+\quad+--C_{-}-$

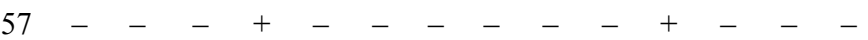

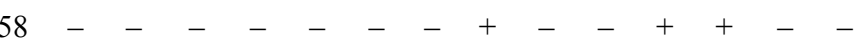

$59-\quad-\quad-\quad-\quad+\quad+-C_{-}-C_{-}-$

$-\quad-\quad-\quad+\quad-\quad-\quad-\quad-\quad-\quad-\quad-\quad-$

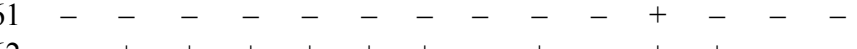

$63-\ldots-\ldots-\ldots+\ldots+\ldots+-$

$\begin{array}{lllllllllllllll}63 & - & - & - & - & - & - & - & + & + & - & + & - & - & - \\ 64 & - & - & - & + & - & + & - & + & + & - & + & - & - & -\end{array}$

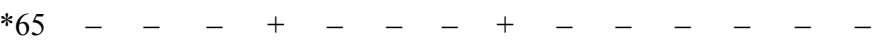

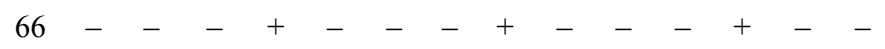

$67+++++++++++$

CIMICOMORPHA

REDUVIIDAE Latreille, 1807

Empicoris Wolff, 1811

culiciformis (DeGeer, 1773) 72

vagabundus (Linnaeus,

1758)

Phymata Latreille, 1802 crassipes (Fabricius, 1775)

Reduvius Fabricius, 1775

personatus (Linnaeus,

1758) 
Table 1. Continued

\begin{tabular}{|c|c|c|c|c|c|c|c|c|c|c|c|c|c|c|c|}
\hline & No. & LG & MG & EL & FL & LF & MF & $\mid \mathrm{NF}$ & EK & FK & LE & ME & $\mathrm{NE}$ & $\mathrm{MD}$ & ND \\
\hline \multicolumn{16}{|l|}{ Pygolampis Germar, 1817} \\
\hline bident & 76 & - & - & - & + & - & + & - & - & + & - & + & + & - & - \\
\hline \multicolumn{16}{|l|}{ Coranus Curtis, 1833} \\
\hline aethiops & $* 77$ & - & - & - & - & + & + & + & + & - & + & - & - & - & - \\
\hline subapterus (DeGeer, 1773) & 78 & + & + & + & + & + & + & - & + & + & + & + & $?$ & + & - \\
\hline \multicolumn{16}{|l|}{ Rhynocoris Hahn, 1834} \\
\hline annulatus (Linnaeus, 1758) & 79 & - & - & - & + & + & - & - & - & - & + & + & + & - & - \\
\hline
\end{tabular}

MICROPHYSIDAE Dohrn, 1859

Loricula Curtis, 1833

pselaphiformis Curtis, 1833

Myrmedobia Bärensprung,

1857

coleoptrata (Fallén, 1807)

distinguenda Reuter, 1884

exilis (Fallén, 1807)

$=$ tenella (Zetterstedt, 1828)

MIRIDAE Hahn, 1833

Bryocoris Fallén, 1829

pteridis (Fallén, 1807)

Monalocoris Dahlbom,

1851

filicis (Linnaeus, 1758)

Dicyphus Fieber, 1858

constrictus (Boheman,

1852)

epilobii Reuter, 1883

globulifer (Fallén, 1829)

stachydis J. Sahlberg, 1878

Bothynotus Fieber, 1864

pilosus Boheman, 1852

Alloeotomus Fieber, 1858

germanicus Wagner, 1939

gothicus (Fallén, 1807)

Deraeocoris Kirschbaum,

1856

morio (Boheman, 1852)

punctulatus (Fallén, 1807)

ruber (Linnaeus, 1758)

scutellaris (Fabricius, 1794)

trifasciatus (Linnaeus,

$$
\text { 1767) }
$$

Adelphocoris Reuter, 1896

lineolatus (Goeze, 1778)

quadripunctatus (Fabricius,

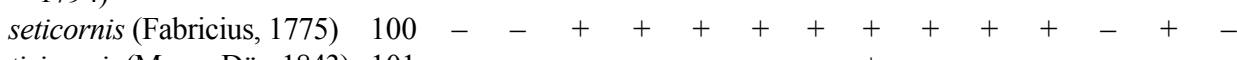

ticinensis (Meyer-Dür, 1843) 101

Continued overleaf 
Table 1. Continued

\begin{tabular}{|c|c|c|c|c|c|c|c|c|c|c|c|c|c|c|c|}
\hline & No. & LG & MG & EL & FL & LF & $\mathrm{MF}$ & $\mathrm{NF}$ & EK & FK & LE & $\mathrm{ME}$ & $\mathrm{NE}$ & $\mathrm{MD}$ & NL \\
\hline \multicolumn{16}{|l|}{ Agnocoris Reuter, 1875} \\
\hline reclai & *102 & - & - & - & - & - & - & - & - & - & - & + & - & - & - \\
\hline rubicundus (Fallén, 1807 & 103 & - & - & - & - & - & - & - & + & + & - & + & - & - & - \\
\hline \multicolumn{16}{|l|}{ Apolygus China, 1941} \\
\hline limbatus (Fallén, 1807) & 104 & - & - & - & - & + & + & - & - & - & - & + & - & - & - \\
\hline lucorum (Meyer-Dür, 1843) & 105 & - & - & + & + & + & - & - & + & + & + & + & - & - & \\
\hline \multicolumn{16}{|l|}{ Calocoris Fieber, 1848} \\
\hline eGeer, & 106 & - & - & + & + & + & + & + & + & + & - & + & + & + & \\
\hline
\end{tabular}
1773)

Camptozygum Reuter, 1896

aequale (Villers, 1789)

Capsodes Dahlbom, 1851

gothicus (Linnaeus, 1758)

Capsus Fabricius, 1803

ater (Linnaeus, 1758) $109-++++++++++++$

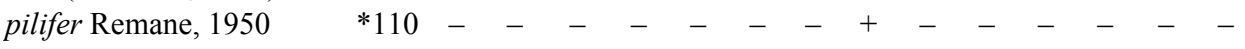

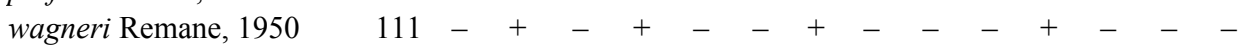

Charagochilus Fieber, 1858

gyllenhalii (Fallén, 1807)

Closterotomus Fieber, 1858

biclavatus (Herrich-

Schaeffer, 1835)

fulvomaculatus (DeGeer,

1773)

norwegicus (Gmelin, 1790)

Dichrooscytus Fieber, 1858

intermedius Reuter, 1885

rufipennis (Fallén, 1807)

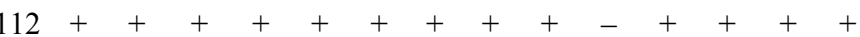

Grypocoris Douglas \&

Scott, 1868

sexguttatus (Fabricius,

1777)

Liocoris Fieber, 1858

tripustulatus (Fabricius,

1781)

Lygocoris Reuter, 1875

contaminatus (Fallén, 1807) $120+-\quad+\quad+\quad+\quad+\quad+++++++$

pabulinus (Linnaeus, 1761) $121-+\quad+\quad+\quad+\quad+\quad+\quad+\quad+\quad+\quad+$

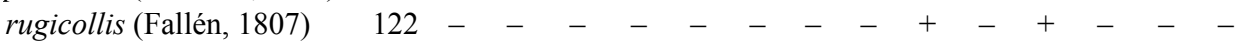

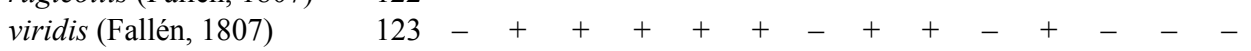

Lygus Hahn, 1833

adspersus (Schilling, 1837) *124 $-+\quad+\quad+\quad+\quad+\quad+\quad+\quad-\quad+\quad-\quad+\quad-$ gemellatus (Herrich-

Schaeffer, 1835)

pratensis (Linnaeus, 1758) $126++++++-+++++++$

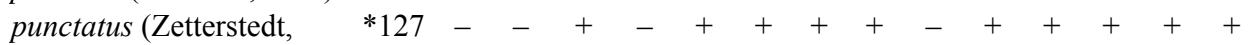

1838) 
Table 1. Continued

\begin{tabular}{|c|c|c|c|c|c|c|c|c|c|c|c|c|c|c|c|}
\hline & No. & LG & MG & EL & FL & $\mathrm{LF}$ & $\mathrm{MF}$ & $\mathrm{NF}$ & EK & FK & LE & $\mathrm{ME}$ & $\mathrm{NE}$ & $\mathrm{MD}$ & \\
\hline rugulipennis Poppius, 1911 & 128 & + & + & - & + & + & + & - & + & + & + & + & + & + & \\
\hline wagneri Remane, 1955 & 129 & - & + & 1 & + & + & + & + & - & - & + & + & + & - & $t$ \\
\hline Miris Fabricius, 1794 & & & & & & & & & & & & & & & \\
\hline striatus (Linnaeus, 1758) & 130 & - & + & - & - & + & + & - & + & + & - & + & - & + & \\
\hline Orthops I & & & & & & & & & & & & & & & \\
\hline basalis (A. Costa, 1853) & 131 & - & + & + & + & + & + & - & + & + & + & + & + & - & \\
\hline campestris (Linnaeus, & 132 & - & + & - & + & + & + & + & - & + & - & + & + & + & \\
\hline kalmii (Linnaeus, 1758) & 133 & - & + & + & + & + & + & - & + & + & - & + & + & + & \\
\hline Pantilius Cur & & & & & & & & & & & & & & & \\
\hline tunicatus (Fabricius, 1781) & 134 & - & - & 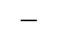 & + & + & + & - & + & + & + & + & + & + & \\
\hline Phytocoris Fallér & & & & & & & & & & & & & & & \\
\hline $\begin{array}{l}\text { dimidiatus Kirschbaum, } \\
\quad 1856\end{array}$ & 135 & - & - & - & - & - & - & - & - & + & + & + & + & + & \\
\hline hirsutulus Flor, 1861 & 36 & - & - & - & - & - & - & - & - & - & + & - & - & - & \\
\hline intricatus Flor, 1861 & 137 & - & + & + & + & - & - & - & - & + & - & - & - & - & \\
\hline longipennis Flor, 1861 & 138 & - & - & + & + & + & + & - & + & + & + & + & + & - & \\
\hline pini Kirschbaum, 1856 & 139 & - & - & - & - & + & - & - & + & - & + & + & + & + & \\
\hline populi (Linnaeus, 1758) & 140 & - & + & - & - & - & - & - & - & + & - & - & - & - & \\
\hline tiliae (Fabricius, 1777) & 141 & - & - & - & - & + & + & - & - & - & - & - & - & - & \\
\hline $\begin{array}{l}\text { ulmi (Linnaeus, 1758) } \\
\text { Pinalitus Kelton, } 1955\end{array}$ & 142 & - & - & - & + & + & - & - & + & - & + & - & - & - & \\
\hline cervinus (Herrich-Schaef & 3 & - & 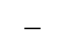 & - & + & + & - & - & + & + & + & + & + & - & \\
\hline
\end{tabular}
1841)

rubricatus (Fallén, 1807) $144-{ }_{-}+{ }_{-}+{ }_{-}+{ }_{-}+{ }_{-}+{ }_{-}+$

Polymerus Hahn, 1831

microphthalmus (Wagner, *145 1951)

nigrita (Fallén, 1807) palustris (Reuter, 1907) tepastus Rinne, 1989

unifasciatus (Fabricius,

1794)

vulneratus (Panzer, 1806)

Rhabdomiris Wagner, 1968

striatellus (Fabricius, 1794)

Stenotus Jakovlev, 1877

binotatus (Fabricius, 1794) *152

Acetropis Fieber, 1858

gimmerthalii (Flor, 1860)

Leptopterna Fieber, 1858

dolabrata (Linnaeus, 1758)

ferrugata (Fallén, 1807)

Megaloceroea Fieber, 1858

recticornis (Geoffroy, 1785) 156

Myrmecoris Gorski, 1852

gracilis (R. F. Sahlberg,

1848)

$\begin{array}{rrrrrrrrrrrrrrr}146 & - & - & + & + & + & + & - & + & + & + & + & - & - & - \\ 147 & - & - & - & + & + & + & - & + & - & + & + & + & - & - \\ * 148 & - & - & - & + & + & - & - & + & + & - & + & - & - & + \\ 149 & - & - & + & + & + & + & - & + & + & + & + & + & - & -\end{array}$

$150-\quad-\quad-\quad-\quad+\quad-\quad+\quad-\quad+-{ }_{-}-{ }_{-}$

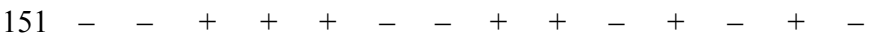

$154-\quad+\quad+\quad+\quad+\quad+\quad+\quad+\quad+\quad+\quad+\quad+$

$155-++\quad+\quad+\quad+\quad+\quad+-$

$156-+\ldots++\quad+\quad+\quad+\quad+\quad+\quad+-$

157

Continued overleaf 
Table 1. Continued

\begin{tabular}{l|l|l|l|l|l|l|l|l|l|l|l|l|l|l|l}
\hline & No. & LG & MG & EL & FL & LF & MF & NF & EK & FK & LE & ME & NE & MD & ND \\
\hline
\end{tabular}

Notostira Fieber, 1858

elongata(Geoffroy, 1758) $158+{ }_{-}+t_{+}++++++++$

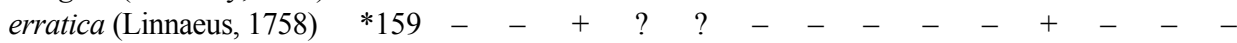

Stenodema Laporte, 1832

calcarata (Fallén, 1807) $160++++++++++++++$

holsata (Fabricius, 1787) $161+++++++_{-}++_{+}++_{-}+$

laevigata (Linnaeus, 1758) $162++++++-+++++++$

trispinosa Reuter, $1904 \quad 163+{ }_{-}{ }_{-}+{ }_{-}+{ }_{-}++_{+}++_{-}+$

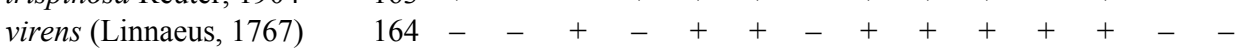

Pithanus Fieber, 1858

maerkeli (Herrich-

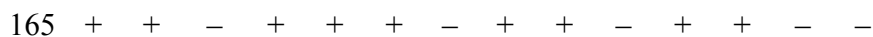

Schaeffer, 1838)

Teratocoris Fieber, 1858

antennatus (Boheman,

1852)

paludum J. Sahlberg, 1870

saundersi Douglas \& Scott,

1869

Trigonotylus Fieber, 1858

caelestialium (Kirkaldy,

ruficornis (Geoffroy, 1785)

Euryopiocoris Reuter, 1875

nitidus (Meyer-Dür, 1843)

Halticus Hahn, 1833

apterus (Linnaeus, 1758)

Labops Burmeister, 1835

sahlbergii (Fallén, 1829)

Orthocephalus Fieber, 1858

brevis (Panzer, 1798)

coriaceus (Fabricius, 1777)

saltator (Hahn, 1835)

vittipennis (Herrich-

Schaeffer, 1835)

Strongylocoris Blanchard,

1840

leucocephalus (Linnaeus,

luridus (Fallén, 1807)

166

167

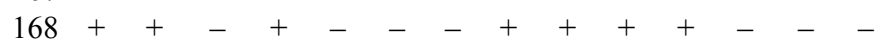

169

170

171

$172+$

173

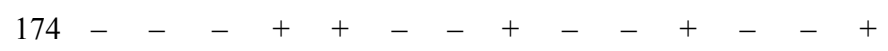

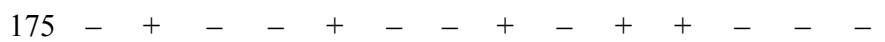

$\begin{array}{lllllllllllllll}175 & - & + & - & - & + & - & - & + & - & + & + & - & - & - \\ 176 & - & + & + & - & + & + & - & + & + & - & + & + & - & -\end{array}$

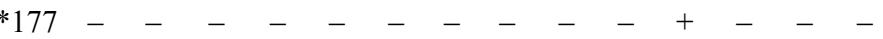

niger (Herrich-Schaeffer,

1835)

steganoides (J. Sahlberg,

Blepharidopterus Kolenati,

1845

angulatus (Fallén, 1807)

diaphanus (Kirschbaum,

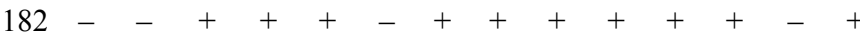
1856)

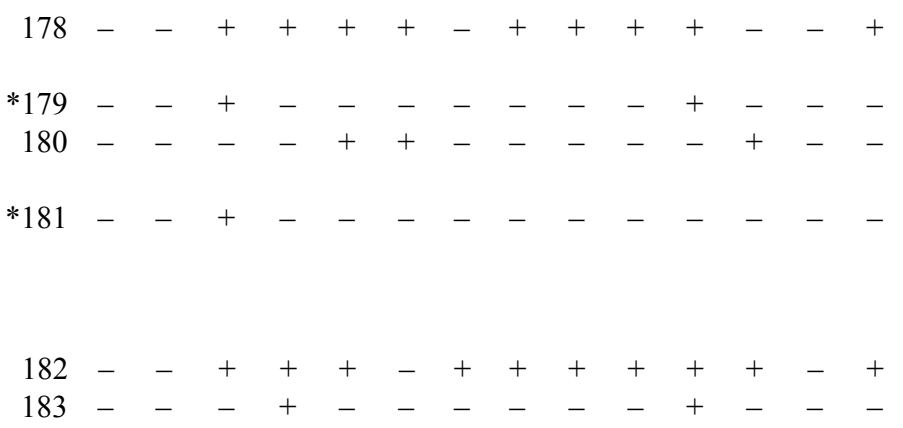


Table 1. Continued

\begin{tabular}{l|l|l|l|l|l|l|l|l|l|l|l|l|l|l|l}
\hline & No. & LG & MG & EL & FL & LF & MF & NF & EK & FK & LE & ME & NE & MD & ND \\
\hline
\end{tabular}

Cyllecoris Hahn, 1834

histrionius (Linnaeus, 1767)

Cyrtorhinus Fieber, 1858

caricis (Fallén, 1807)

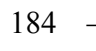

Dryophilocoris Reuter, 1875

flavoquadrimaculatus

(DeGeer, 1773)

Fieberocapsus Carvalho \&

Southwood 1955

flaveolus (Reuter, 1870)

Globiceps Lepeletier \&

Serville, 1825

flavomaculatus (Fabricius, 1794)

fulvicollis Jakovlev, 1877

salicicola Reuter, 1880

sphegiformis (Rossi, 1790)

Heterocordylus Fieber,

1858

erythrophthalmus (Hahn, 1831)

Malacocoris Fieber, 1858 chlorizans (Panzer, 1794)

Mecomma Fieber, 1858

ambulans (Fallén, 1807)

dispar (Boheman, 1852)

Orthotylus Fieber, 1858

bilineatus (Fallén, 1807)

ericetorum (Fallén, 1807)

flavosparsus (C. R.

Sahlberg, 1842)

marginalis Reuter, 1883

nassatus (Fabricius, 1787)

prasinus (Fallén, 1826)

tenellus (Fallén, 1807)

virens (Fallén, 1807)

viridinervis (Kirschbaum, *204

1856)

Pilophorus Hahn, 1826

cinnamopterus

(Kirschbaum, 1856)

clavatus (Linnaeus, 1767)

confusus (Kirschbaum,

206

1856)

Hallodapus Fieber, 1858

rufescens (Burmeister,

1835)

$\begin{array}{llllllllllllllllll}193 & - & - & + & + & + & + & - & + & + & - & + & - & - & - \\ 194 & - & + & + & - & + & + & - & + & + & + & + & + & - & - \\ 195 & - & - & - & - & + & - & - & - & - & + & - & - & - & - \\ 196 & - & - & - & - & - & - & - & - & - & - & + & - & + & - \\ 197 & - & - & + & + & + & + & - & + & + & + & + & - & - & - \\ 198 & + & + & + & + & + & + & - & + & + & + & + & + & + & -\end{array}$

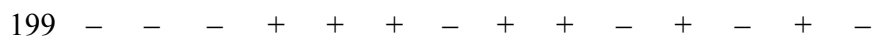

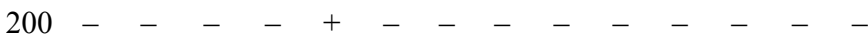

$201-{ }_{-}+\mathrm{C}_{-}-\mathrm{C}_{-}+\mathrm{C}_{-}-\mathrm{C}_{-}$

$202-\mathrm{C}_{2}-\mathrm{C}_{-}-\mathrm{C}_{-}+\mathrm{C}_{-}-\mathrm{C}_{-}$

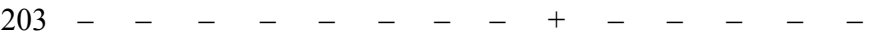

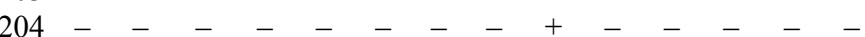


Table 1. Continued

\begin{tabular}{l|l|l|l|l|l|l|l|l|l|l|l|l|l|l|l}
\hline & No. & LG & MG & EL & FL & LF & MF & NF & EK & FK & LE & ME & NE & MD & ND \\
\hline
\end{tabular}

Systellonotus Fieber, 1858

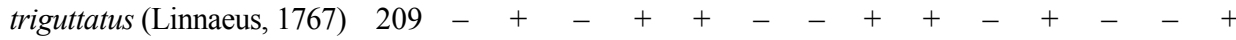
Amblytylus Fieber, 1858

nasutus (Kirschbaum, 1856) $210-{ }_{-} \quad+\quad+\quad+\quad+\quad-\quad+\quad-\quad+\quad-\quad-$

Atractotomus Fieber, 1858

magnicornis (Fallén, 1807) $211-c_{1} \quad-\quad-\quad-\quad+\quad-\quad-\quad-\quad-\quad+\quad+\quad-\quad-$

mali (Meyer-Dür, 1843)

Brachyarthrum Fieber,

1858

limitatum Fieber, 1858

Campylomma Reuter, 1878

verbasci (Meyer-Dür, 1843) 214

Chlamydatus Curtis, 1833

pulicarius (Fallén, 1807)

pullus (Reuter, 1870)

saltitans (Fallén, 1807)

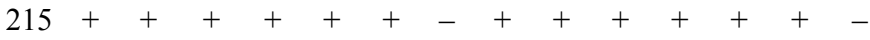

$216++\quad+\quad+\quad+\quad-\quad+\quad+\quad+\quad-\quad-$

Compsidolon Reuter, 1899

salicellum (Herrich-

Schaeffer, 1841)

Conostethus Fieber, 1858

roseus (Fallén, 1807)

Criocoris Fieber, 1858

crassicornis (Hahn, 1834)

quadrimaculatus (Fallén,

1807)

Europiella Reuter, 1909

albipennis (Fallén, 1829)

artemisiae (Becker, 1864)

Hoplomachus Fieber, 1858

thunbergii (Fallén, 1807)

Lopus Hahn, 1833

decolor (Fallén, 1807)

Megalocoleus Reuter, 1890

molliculus (Fallén, 1807) 226

tanaceti (Fallén, 1807)

$=$ pilosus $($ Schrank, 1801)

Monosynamma Scott, 1864

bohemanni (Fallén, 1829)

$=$ nigritula $($ Zetterstedt, 1838)

Oncotylus Fieber, 1858

viridiflavus (Goeze, 1778)

Orthonotus Stephens, 1829

rufifrons (Fallén, 1807)

$217+-+\quad+\quad+\quad+\quad+\quad+\quad-\quad-$

218

219

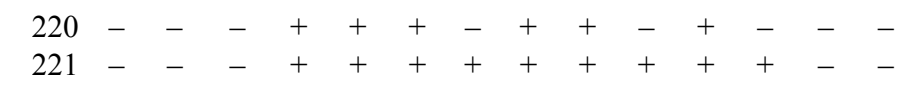

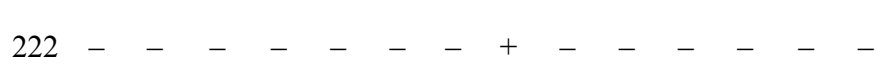

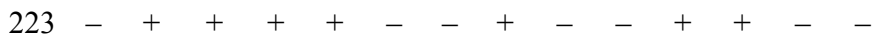

224

225

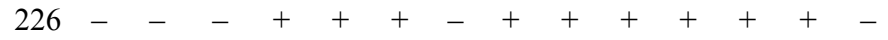

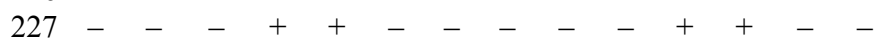

228

Phoenicocoris Reuter, 1875

modestus (Meyer-Dür,

1843)

obscurellus (Fallén, 1829)

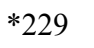


Table 1. Continued

\begin{tabular}{l|l|l|l|l|l|l|l|l|l|l|l|l|l|l|l}
\hline & No. & LG & MG & EL & FL & LF & MF & NF & EK & FK & LE & ME & NE & MD & ND \\
\hline
\end{tabular}

Phylus Hahn, 1831

coryli (Linnaeus, 1758) 233

melanocephalus (Linnaeus, 234

1767)

Placochilus Fieber, 1858

seladonicus (Fallén, 1807) $235-c_{-}+c_{-}+c_{-}+t_{+}++_{+}+$

Plagiognathus Fieber, 1858

arbustorum (Fabricius, $236++++++-+++++++$ 1794)

chrysanthemi (Wolff, 1804) 237

Plesiodema Reuter, 1875

pinetella (Zetterstedt, 1828) 238

Psallus Fieber, 1858

ambiguus (Fallén, 1807) $239-c_{-}+c_{-}+t_{-}+t_{+}+{ }_{-}+$

betuleti (Fallén, 1826) $240+-{ }_{-}+{ }_{-}+-+++++-{ }_{-}+$

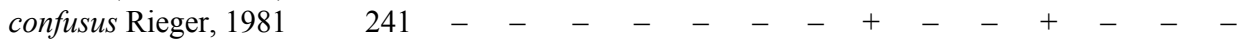

falleni Reuter, $1883 \quad 242$

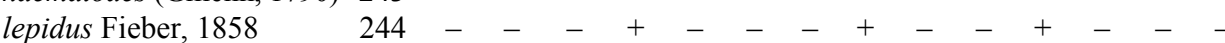

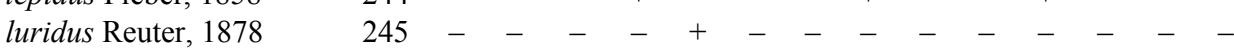

mollis (Mulsant \& Rey, 246

1852)

perrisi (Mulsant \& Rey,

1852)

salicis (Kirschbaum, 1856) 248

$=$ alnicola Douglas \&

Scott, 1871

$=$ scholtzi Fieber, 1861

variabilis (Fallén, 1807) 249

varians (Herrich-Schaeffer, 250

1841)

Salicarus Kerzhner, 1962

roseri (Herrich-Schaeffer, 251

1838)

Tytthus Fieber, 1864

pubescens (Knight, 1931) 252

$=$ geminus (Flor, 1860)

pygmaeus (Zetterstedt,

1838)

TINGIDAE Laporte, 1832

Acalypta Westwood, 1840

carinata (Panzer, 1806)

gracilis (Fieber, 1844)

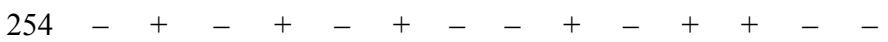

marginata (Wolff, 1804)

nigrina (Fallén, 1807)

$255+$

parvula (Fallén, 1807)

257

platycheila (Fieber, 1844)

258

259

-
-
+
-
-

$\begin{array}{ll}- & + \\ - & + \\ + & + \\ + & + \\ + & +\end{array}$

+
+
+
+
+

$+$

$+++t_{-}+$

$+-+-\quad+\quad-\quad-$

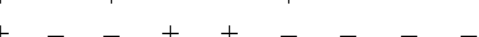

$-\quad+\quad+\quad+\quad+\quad+$

Continued overleaf 
Table 1. Continued

\begin{tabular}{|c|c|c|c|c|c|c|c|c|c|c|c|c|c|c|c|}
\hline & No. & $\mathrm{LG}$ & MG & EL & FL & $\mathrm{LF}$ & MF & $\mathrm{NF}$ & EK & FK & LE & $\mathrm{ME}$ & $\mathrm{NE}$ & $\mathrm{MD}$ & ND \\
\hline \multicolumn{16}{|l|}{ Agramma Stephens, 1829} \\
\hline femorale Thomson, 1871 & 260 & - & + & + & + & + & + & - & + & + & + & + & + & - & - \\
\hline tropidopterum Flor, 1860 & 261 & - & - & - & - & + & + & - & - & - & - & + & + & - & - \\
\hline \multicolumn{16}{|l|}{ Catoplatus Spinola, 1837} \\
\hline fabricii (Stål, 1868) & 262 & - & - & - & + & - & - & - & + & + & - & + & - & - & - \\
\hline \multicolumn{16}{|l|}{ Derephysia Spinola, 1837} \\
\hline cristata (Panzer, 1806) & 263 & - & - & - & - & - & - & - & + & - & - & - & - & - & - \\
\hline foliacea (Fallén, 18 & 264 & - & + & - & + & + & + & - & + & + & - & + & - & - & - \\
\hline \multicolumn{16}{|l|}{ Dictyla Stål, 1874} \\
\hline $\begin{array}{l}\text { convergens (Herrich- } \\
\text { Schaeffer, 1835) }\end{array}$ & 265 & + & + & - & - & - & - & - & - & - & - & + & + & - & - \\
\hline echii (Schrank, 1 & 266 & - & - & + & - & - & - & - & + & - & - & + & - & - & - \\
\hline $\begin{array}{l}\text { lupuli (Herrich-Schaeffer, } \\
\text { 1837) }\end{array}$ & 267 & - & - & - & - & - & - & - & - & - & - & + & + & - & - \\
\hline \multicolumn{16}{|l|}{ Galeatus Curtis, 1833} \\
\hline $\begin{array}{l}\text { spinifrons (Fallén, 1807) } \\
=\text { affinis sensu Flor, } 1860 \\
\text { non Herrich-Schaeffer, } \\
1835\end{array}$ & 268 & - & - & - & - & + & - & - & + & - & - & + & - & - & - \\
\hline \multicolumn{16}{|l|}{ Kalama Puton, 1876} \\
\hline tricornis (Schrank, 1801) & 269 & + & + & - & + & + & - & - & + & + & - & - & - & - & - \\
\hline \multicolumn{16}{|l|}{ Physatocheila Fieber, 1844} \\
\hline costata (Fabricius, 1794) & 270 & - & - & - & + & + & + & + & + & - & - & + & + & - & - \\
\hline \multicolumn{16}{|l|}{ Stephanitis Stål, 1873} \\
\hline oberti (Kolenati, 1856) & 271 & - & - & + & - & + & + & + & + & - & - & + & + & - & - \\
\hline $\begin{array}{l}\text { Tingis Fabricius, } 1803 \\
\text { cardui (Linnaeus, 1758) }\end{array}$ & 272 & - & + & + & + & + & + & - & + & + & - & + & - & - & - \\
\hline
\end{tabular}

NABIDAE A. Costa, 1853

Himacerus Wolff, 1811

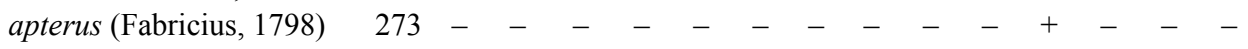

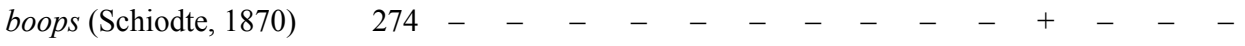

Nabis Latreille, 1802

brevis Scholtz, $1847275++++++++++++++$

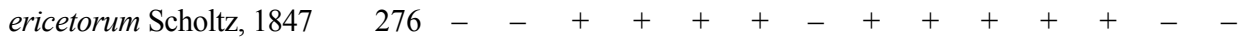

$\begin{array}{lllllllllllllllll}\text { ferus (Linnaeus, 1758) } & 277 & + & + & - & + & + & + & + & + & + & + & + & + & - & - \\ \text { flavomarginatus Scholtz, } & 278 & + & + & + & + & + & + & + & + & + & + & + & + & + & +\end{array}$

1847

limbatus Dahlbom, $1851279++\quad+\quad+\quad+\quad++++++\quad+\quad+$

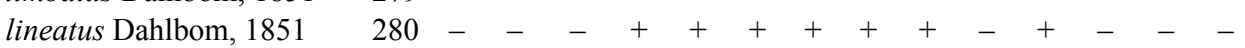

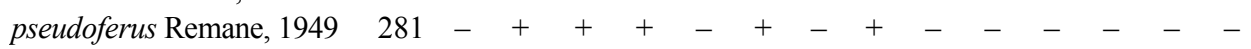

punctatus A. Costa, 1847 *282 $\quad-\quad-\quad-\quad-\quad-\quad-\quad-\quad-\quad-\quad-\quad+\quad-\quad-$

rugosus (Linnaeus, 1758) $283-{ }_{-}+{ }_{+}+{ }_{-}+{ }_{+}+{ }_{+}+{ }_{-}$

ANTHOCORIDAE Fieber, 1836

Acompocoris Reuter, 1875

pygmaeus (Fallén, 1807) 
Table 1. Continued

\begin{tabular}{|c|c|c|c|c|c|c|c|c|c|c|c|c|c|c|c|}
\hline & No. & LG & $\mathrm{MG}$ & EL & FL & LF & MF & $\mathrm{NF}$ & EK & FK & LE & ME & NE & MD & ND \\
\hline \multicolumn{16}{|l|}{ Anthocoris Fallén, 1814} \\
\hline confusus Reuter, 1884 & 285 & - & - & - & + & - & + & - & + & - & - & - & + & - & - \\
\hline $\begin{array}{l}\text { gallarumulmi (DeGeer, } \\
\text { 1773) }\end{array}$ & 286 & - & - & - & + & - & - & - & + & - & + & + & + & - & - \\
\hline nemoralis (Fabricius, 1794) & 287 & - & + & - & - & + & - & - & - & + & - & + & - & - & - \\
\hline nemorum (Linnaeus, 1761) & 288 & + & + & + & + & + & + & + & + & + & + & + & + & + & - \\
\hline pilosus (Jakovlev, 1877) & *289 & - & - & - & - & + & - & - & - & - & - & - & - & - & - \\
\hline simulans Reuter, 1884 & 290 & - & - & - & - & - & - & - & + & - & - & + & - & - & + \\
\hline \multicolumn{16}{|l|}{ Elatophilus Reuter, 1884} \\
\hline stigmatellus (Zetterstedt, & *291 & - & - & - & - & - & - & - & - & - & - & - & + & - & - \\
\hline
\end{tabular}
1838)

Temnostethus Fieber, 1860 gracilis Horváth, 1907 pusillus (Herrich-Schaeffer, 293

Tetraphleps Fieber, 1860 aterrimus (J. Sahlberg, 1878)

bicuspis (Herrich-Schaeffer, 295

Orius Wolff, 1811

laticollis (Reuter, 1884)

minutus (Linnaeus, 1758)

niger (Wolff, 1811)

Lyctocoris Hahn, 1836

campestris (Fabricius, 1794) 299

Scoloposcelis Fieber, 1864

obscurella (Zetterstedt,

pulchella (Zetterstedt, 1838) *301

Xylocoris Dufour, 1831

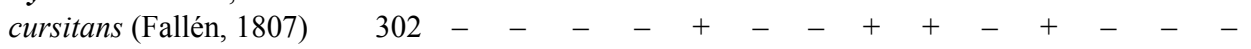

formicetorum (Boheman, *303

1844)

galactinus (Fieber, 1836)

Amphiareus Distant, 1904

obscuripes (Poppius, 1909) *305

CIMICIDAE Latreille, 1802

Cimex Linnaeus, 1758

lectularius Linnaeus, 1758306

Oeciacus Stål, 1873

hirundinis (Lamarck, 1816) 307

PENTATOMOMORPHA

ARADIDAE Brullé, 1836

Aradus Fabricius, 1803

aterrimus Fieber, 1864

Continued overleaf 
Table 1. Continued

\begin{tabular}{|c|c|c|c|c|c|c|c|c|c|c|c|c|c|c|c|}
\hline & No. & $\mathrm{LG}$ & $\mathrm{MG}$ & EL & FL & LF & $\mathrm{MF}$ & $\mathrm{NF}$ & EK & FK & LE & $\mathrm{ME}$ & $\mathrm{NE}$ & MD & ND \\
\hline betulae (Linnaeus, 1758) & 309 & - & - & - & - & + & - & - & + & - & - & + & + & - & - \\
\hline betulinus Fallén, 1829 & 310 & - & + & - & - & - & - & - & - & - & - & - & - & - & - \\
\hline $\begin{array}{l}\text { cinnamomeus (Panzer, } \\
\text { 1806) }\end{array}$ & 311 & - & - & + & + & - & + & - & + & + & - & + & + & - & - \\
\hline corticalis (Linnaeus, 1758) & 312 & - & - & + & - & - & - & - & + & - & - & + & + & - & - \\
\hline depressus (Fabricius, 1794) & 313 & - & + & - & - & + & + & - & - & + & - & + & - & - & - \\
\hline lugubris Fallén, 1807 & 314 & - & - & - & - & - & - & + & - & - & - & + & - & - & - \\
\hline truncatus Fieber, 1861 & 315 & - & - & - & - & + & - & - & - & - & - & - & - & - & - \\
\hline
\end{tabular}

PIESMATIDAE Amyot \& Serville, 1843

Parapiesma Péricart, 1974

quadratum (Fieber, 1844)

316

Piesma Lepeletier \&

Serville, 1828

capitatum (Wolff, 1804) $317-c_{-} \quad-\quad-\quad+\quad-\quad+\quad+\quad-\quad+\quad+\quad-$

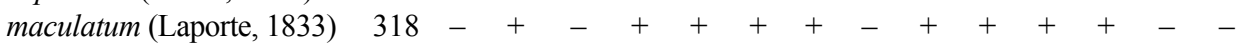

BERYTIDAE Fieber, 1851

Neides Latreille, 1802

tipularius (Linnaeus, 1758) *319

Berytinus Kirkaldy, 1900

clavipes (Fabricius, 1775)

crassipes (Herrich-

Schaeffer, 1835)

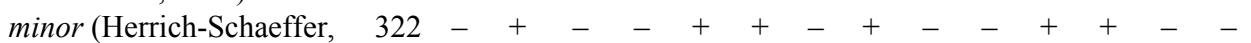

1835)

montivagus (Meyer-Dür,

1841)

signoreti (Fieber, 1859)

Metatropis Fieber, 1859

rufescens (Herrich-

Schaeffer, 1835)

$320 \quad-\quad-\quad-\quad+\quad+\quad+\quad-\quad+\quad+\quad-\quad+\quad+\quad-$

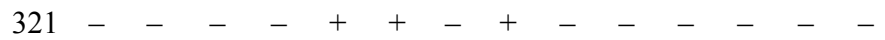

*323- - - ?

324

325

LYGAEIDAE Schilling, 1829

Lygaeus Fabricius, 1794

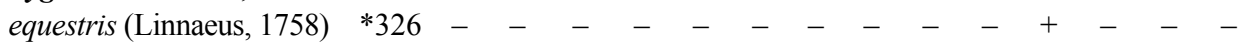

Nithecus Horváth, 1890

jacobaeae (Schilling, 1829) $327-{ }_{-}++_{+}+{ }_{-}+++{ }_{-}+$

Nysius Dallas, 1852

ericae (Schilling, 1829)

helveticus (Herrich-

Schaeffer, 1850)

thymi (Wolff, 1804)

Ortholomus Stål, 1872

punctipennis (Herrich-

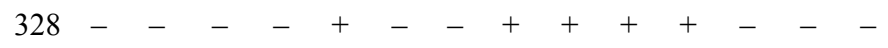

$329-\ldots+\ldots+\ldots+\ldots+\ldots$

$330++\quad+\quad+\quad+\quad-\quad+\quad+\quad+\quad+\quad-\quad+$

Schaeffer, 1838)

Kleidocerys Stephens, 1829

resedae (Panzer, 1797)

$332++\quad+\quad+\quad+\quad+\quad+\quad+\quad+\quad+\quad-$ 
Table 1. Continued

\begin{tabular}{l|l|l|l|l|l|l|l|l|l|l|l|l|l|l|l}
\hline & No. & LG & MG & EL & FL & LF & MF & NF & EK & FK & LE & ME & NE & MD & ND \\
\hline
\end{tabular}

Cymus Hahn, 1831

aurescens Distant, 1883 obliquus Horváth, 1888
$\quad=$ o

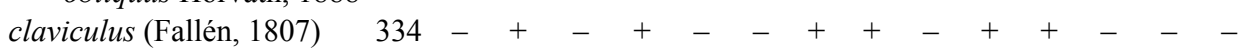

glandicolor Hahn, $1832335++++++++++++++++$

Ischnodemus Fieber, 1837

sabuleti (Fallén, 1826)

Geocoris Fallén, 1814

ater (Fabricius, 1787)

dispar (Waga, 1839)

$336++\quad++$

grylloides (Linnaeus, 1761)

lapponicus Zetterstedt, 1838

Chilacis Fieber, 1864

typhae (Perris, 1857)

Heterogaster Schilling,

1829

urticae (Fabricius, 1775)

Camptotelus Fieber, 1860

lineolatus (Schilling, 1829)

Macroplax Fieber, 1860

preyssleri (Fieber, 1837)

Philomyrmex R. F.

Sahlberg, 1848

insignis R. F. Sahlberg, 1848345

Tropidophlebia Kerzhner,

1964

costalis (Herrich-Schaeffer, *346

1850)

Tropistethus Fieber, 1860

holosericeus (Scholtz, 1846) 347

Drymus Fieber, 1860

brunneus (R. F. Sahlberg, 348 1848)

pilicornis (Mulsant \& Rey, 349

1852)

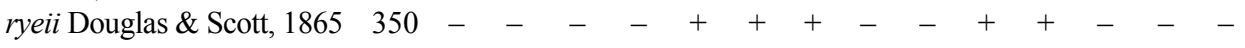

sylvaticus (Fabricius, 1775) $351-{ }_{-}-+++-{ }_{-}+{ }_{+}+{ }_{+}+{ }_{-}$

Eremocoris Fieber, 1860

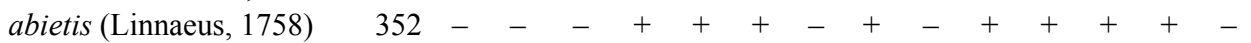

plebejus (Fallén, 1807)

Gastrodes Westwood, 1840

abietum Bergroth, $1914 \quad 354-{ }_{-}+{ }_{-}+{ }_{-}-+{ }_{-}+{ }_{-}+{ }_{-}+$

grossipes (DeGeer, 1773) $355-{ }_{-}+{ }_{-}+-{ }_{-}+{ }_{-}+{ }_{-}-{ }_{-}$

Ischnocoris Fieber, 1860

angustulus (Boheman,

1852)

Continued overleaf 
Table 1. Continued

\begin{tabular}{l|l|l|l|l|l|l|l|l|l|l|l|l|l|l|l}
\hline & No. & LG & MG & EL & FL & LF & MF & NF & EK & FK & LE & ME & NE & MD & ND \\
\hline
\end{tabular}

Lamproplax Douglas \& Scott, 1868 picea (Flor, 1860) 357 Scolopostethus Fieber, 1860

affinis (Schilling, 1829) decoratus (Hahn, 1833) pictus (Schilling, 1829) pilosus Reuter, 1875 thomsoni Reuter, 1875 Aphanus Laporte, 1833 rolandri (Linnaeus, 1758)

Emblethis Fieber, 1860 verbasci (Fabricius, 1803)

Gonianotus Fieber, 1860 marginepunctatus (Wolff, 1804)

Macrodema Fieber, 1860 micropterum (Curtis, 1836) Pionosomus Fieber, 1860 varius (Wolff, 1804)

Pterotmetus Amyot \& Serville, 1843

staphyliniformis (Schilling, 1829)

Trapezonotus Fieber, 1860 anorus (Flor, 1860) arenarius (Linnaeus, 1758) desertus Seidenstücker,

1951

Megalonotus Fieber, 1860 antennatus (Schilling, 1829) $372-+-{ }_{-}++-++-+-$ chiragra (Fabricius, 1794) $373++-+_{+}+++{ }_{-}+{ }_{-}+-$ Sphragisticus Stål, 1872 nebulosus (Fallén, 1807) Ligyrocoris Stål, 1872 sylvestris (Linnaeus, 1758) Pachybrachius Hahn, 1826 fracticollis (Schilling, 1829) luridus Hahn, 1826

Plinthisus Stephens, 1829 pusillus (Scholtz, 1847) Graptopeltus Stål, 1872 lynceus (Fabricius, 1775) Peritrechus Fieber, 1860 angusticollis (R. F. Sahlberg, 1848)

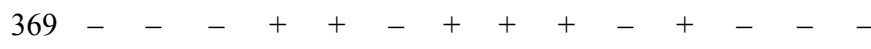

$370++++++++\infty+-$

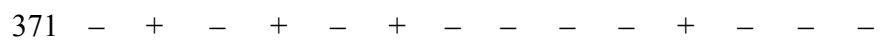
365

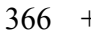


Table 1. Continued

\begin{tabular}{|c|c|c|c|c|c|c|c|c|c|c|c|c|c|c|c|}
\hline & No. & $\mathrm{LG}$ & MG & EL & FL & LF & MF & $\mathrm{NF}$ & EK & FK & LE & $\mathrm{ME}$ & $\mathrm{NE}$ & $\mathrm{MD}$ & ND \\
\hline onv & 381 & - & + & - & + & + & - & - & + & + & + & - & - & - & - \\
\hline \multicolumn{16}{|l|}{$=$ distinguendus (Flor, 1860) } \\
\hline geniculatus (Hahn, 1832) & 382 & - & - & - & + & + & - & - & + & + & - & + & + & - & - \\
\hline nubilus (Fallén, 1807) & 383 & + & + & - & + & + & - & - & + & + & - & - & - & - & - \\
\hline \multicolumn{16}{|l|}{ Raglius Stål, 1872} \\
\hline $\begin{array}{l}\text { alboacuminatus (Goeze, } \\
1778 \text { ) }\end{array}$ & *384 & - & - & - & - & - & - & - & - & - & - & + & - & - & - \\
\hline \multicolumn{16}{|l|}{$\begin{array}{l}\text { Rhyparochromus Hahn, } \\
1826\end{array}$} \\
\hline $\begin{array}{l}\text { pini (Linnaeus, 1758) } \\
\text { Acompus } \text { Fieber, } 1860\end{array}$ & 385 & - & - & + & + & + & + & - & + & + & + & + & + & - & - \\
\hline rufipes (Wolff, 1804) & 386 & - & - & + & + & + & + & - & + & + & + & + & - & - & - \\
\hline \multicolumn{16}{|l|}{$\begin{array}{l}\text { Stygnocoris Douglas \& } \\
\text { Scott, } 1865\end{array}$} \\
\hline fuligineus (Geoffroy, 1785) & 387 & - & - & + & - & + & - & - & - & - & - & + & + & - & - \\
\hline $\begin{array}{l}\text { pygmaeus (R. F. Sahlberg, } \\
1848)\end{array}$ & 388 & - & + & + & + & - & - & - & + & + & + & - & - & - & - \\
\hline rusticus (Fallén, 1807) & 389 & - & - & - & + & + & + & + & + & + & + & + & + & - & - \\
\hline $\begin{array}{l}\text { sabulosus (Schilling, 1829) } \\
=\text { pedestris (Fallén, 1807) }\end{array}$ & 390 & - & + & + & + & + & + & - & + & + & + & + & + & - & - \\
\hline
\end{tabular}

PYRRHOCORIDAE Amyot \& Serville, 1843

Pyrrhocoris Fallén, 1814

apterus (Linnaeus, 1758)

COREIDAE Leach, 1815

Coreus Fabricius, 1794

marginatus (Linnaeus,

Spathocera Stein, 1860

dahlmannii (Schilling,

laticornis (Schilling, 1829) *394

Bathysolen Fieber, 1860

nubilus (Fallén, 1807)

Ulmicola Kirkaldy, 1909

spinipes (Fallén, 1807)

Nemocoris R. F. Sahlberg,

1848

falleni R. F. Sahlberg, 1848

*391

Coriomeris Westwood,

1842

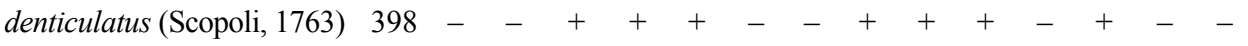

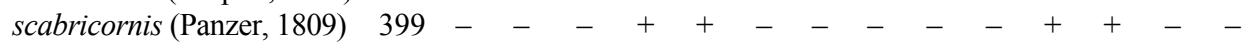

RHOPALIDAE Amyot \& Serville, 1843

Corizus Fallén, 1814

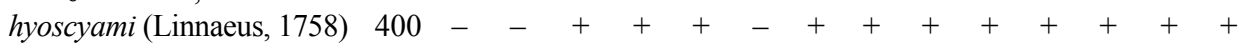

Rhopalus Schilling, 1827

Continued overleaf 
Table 1. Continued

\begin{tabular}{|c|c|c|c|c|c|c|c|c|c|c|c|c|c|c|c|}
\hline & No. & $\mathrm{LG}$ & MG & EL & FL & $\mathrm{LF}$ & MF & $\mathrm{NF}$ & EK & FK & LE & ME & NE & MD & ND \\
\hline nacul & 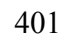 & + & - & + & + & + & + & + & + & + & + & + & + & + & + \\
\hline $\begin{array}{l}\text { parumpunctatus Schilling, } \\
1829\end{array}$ & 402 & + & - & + & + & + & + & - & + & + & + & + & + & - & - \\
\hline subrufus (Gmelin, 1790) & 403 & - & - & + & + & + & + & - & + & + & + & + & + & - & + \\
\hline Stictor & & & & & & & & & & & & & & & \\
\hline butilon $(\mathrm{R}$ & 404 & + & - & + & + & + & + & + & + & + & + & + & + & + & + \\
\hline $\begin{array}{l}\text { crassicornis } \\
1758)\end{array}$ & 405 & + & + & + & + & + & + & + & + & + & + & + & + & + & - \\
\hline $\begin{array}{l}\text { punctatonervosus (Goeze, } \\
1778 \text { ) }\end{array}$ & 406 & - & - & - & 1 & + & + & + & - & - & + & + & + & + & + \\
\hline Chor & & & & & & & & & & & & & & & \\
\hline $\begin{array}{l}\text { schilling } \\
\text { Myrmu: }\end{array}$ & 407 & - & - & + & + & - & - & - & + & + & - & - & - & - & - \\
\hline nirifo & 408 & 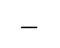 & - & + & + & + & + & + & + & + & + & + & + & - & + \\
\hline
\end{tabular}

ALYDIDAE Amyot \& Serville, 1843

Alydus Fabricius, 1803

calcaratus (Linnaeus, 1758) 409

ACANTHOSOMATIDAE Signoret, 1863

Acanthosoma Curtis, 1824

haemorrhoidale (Linnaeus, $410-{ }_{-}+{ }_{-}+{ }_{-}-{ }_{-}+{ }_{-}+{ }_{+}+$ 1758)

Cyphostethus Fieber, 1860

tristriatus (Fabricius, 1787)

Elasmostethus Fieber, 1860

brevis Lindberg, 1934

interstinctus (Linnaeus,

$$
\text { 1758) }
$$

minor Horváth, 1899

Elasmucha Stål, 1864

ferrugata (Fabricius, 1787) $415-{ }_{-}+t_{-}+-\quad+-{ }_{-}+-\quad+-$

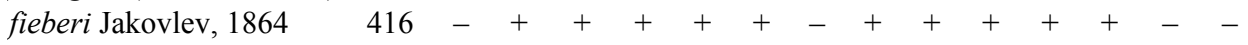

grisea (Linnaeus, 1758) $417++\quad+\quad+\quad+++++++++$

CYDNIDAE Billberg, 1820

Legnotus Schiodte, 1848

picipes (Fallén, 1807)

Adomerus Mulsant \& Rey,

1866

biguttatus (Linnaeus, 1758) 419

Sehirus Amyot \& Serville,

1843

luctuosus Mulsant \& Rey, 420

1866

Tritomegas Amyot \&

Serville, 1843

bicolor (Linnaeus, 1758) 
Table 1. Continued

\begin{tabular}{l|l|l|l|l|l|l|l|l|l|l|l|l|l|l|l}
\hline & No. & LG & MG & EL & FL & LF & MF & NF & EK & FK & LE & ME & NE & MD & ND \\
\hline
\end{tabular}

THYREOCORIDAE Amyot \& Serville, 1843

Thyreocoris Schrank, 1801 scarabaeoides (Linnaeus, 1758)

SCUTELLERIDAE Leach, 1815

Eurygaster Laporte, 1832

maura (Linnaeus, 1758)

testudinaria (Geoffroy,

$\begin{array}{lllllllllllllll}423 & - & - & - & - & - & - & - & - & - & - & + & - & - & - \\ 424 & - & - & + & + & + & + & + & + & + & - & + & + & + & -\end{array}$

1785)

Odontoscelis Laporte, 1832

fuliginosa (Linnaeus, 1761)

Phimodera Germar, 1839

humeralis (Dalman, 1823)

PENTATOMIDAE Leach, 1815

Dybowskyia Jakovlev, 1876

reticulata Dallas, 1851

Graphosoma Laporte, 1832

lineatum (Linnaeus, 1758) *428

Sciocoris Fallén, 1829

cursitans (Fabricius, 1794) 429

macrocephalus Fieber, $1851 * 430$

microphthalmus Flor, 1860431

umbrinus (Wolff, 1804) 432

Aelia Fabricius, 1803

acuminata (Linnaeus, 1758) $433-\quad+\quad+\quad+\quad+\quad+\quad+\quad+\quad+\quad+\quad+-$

klugii Hahn, 1831

Neottiglossa Kirby, 1837

pusilla (Gmelin, 1790)

Eysarcoris Hahn, 1834

aeneus (Scopoli, 1763)

Rubiconia Dohrn, 1860

intermedia (Wolff, 1811)

Antheminia Mulsant \&

Rey, 1866

aliena (Reuter, 1891)

$434-\mathrm{C}_{-}+\mathrm{C}_{-}+\mathrm{C}_{-}+\mathrm{C}_{-}$

Carpocoris Kolenati, 1846

fuscispinus (Boheman, 1850)

purpureipennis (DeGeer, 1773)

Chlorochroa Stål, 1872

$\begin{array}{lllllllllllllllllll}\text { juniperina (Linnaeus, 1758) } & 441 & + & + & + & + & + & + & - & + & + & - & + & + & - & - \\ \text { pinicola Mulsant \& Rey, } & 442 & - & - & - & - & + & + & - & - & - & - & + & + & - & -\end{array}$ 1852

Dolycoris Mulsant \& Rey, 1866

baccarum (Linnaeus, 1758)

\section{5}

$* 436$

437

$* 438$

439

440 
Table 1. Continued

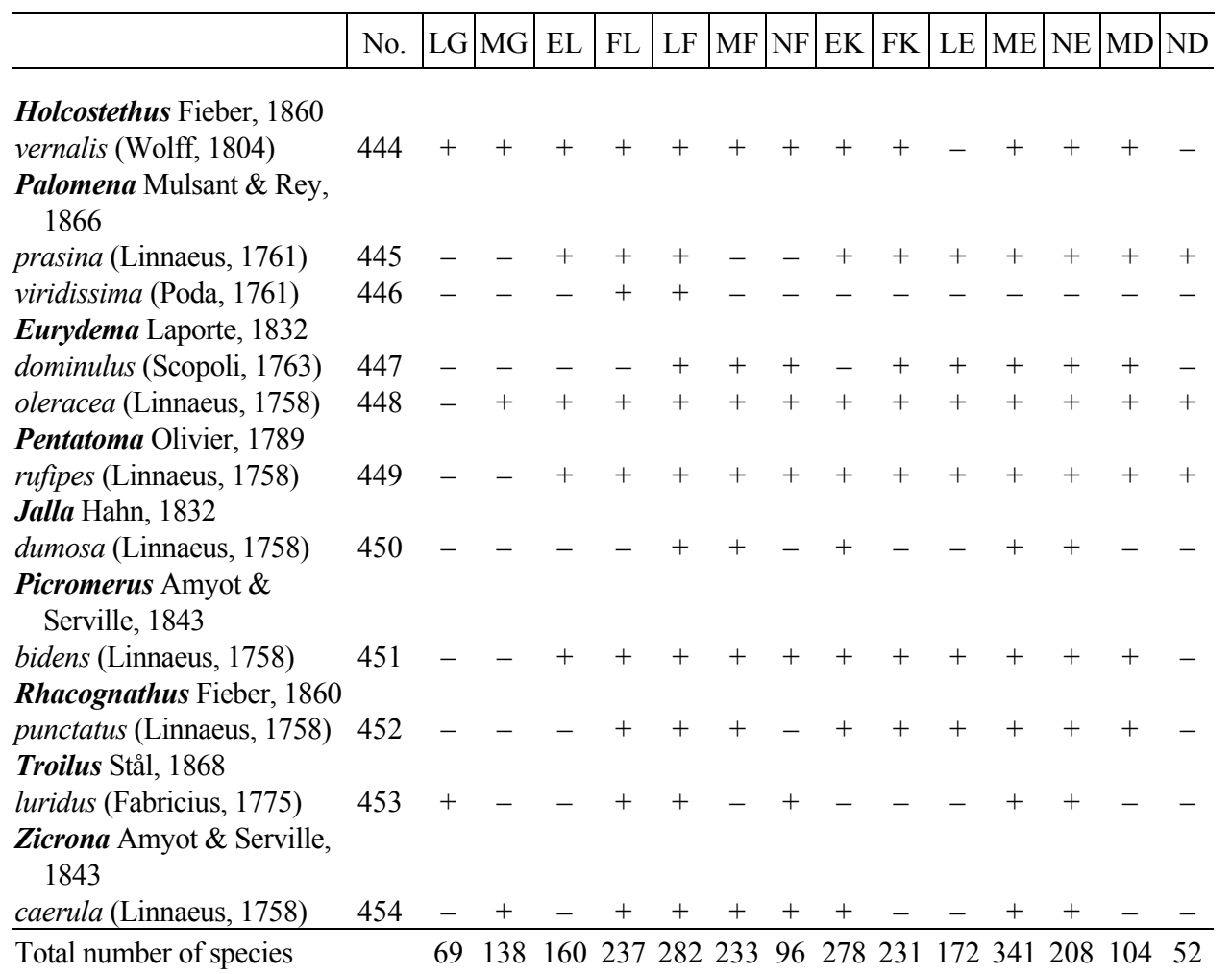

\section{Remarks to the species marked with an asterisk (*)}

8 buenoi Only recorded from MF 24 Roosna-Alliku, Allikjärve 12.v.1982, 7 ơ 10 ๆ (Valk 1984).

21 griseola See Coulianos (2003).

26 cavifrons See Coulianos (2003).

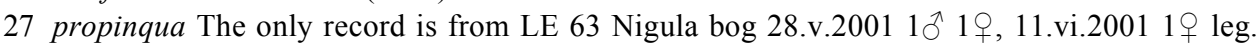
M. Smits det. C.-C. Coulianos. This record will be published in full by G. A. van Duinen (in prep.).

29 wollastoni The only record is from LE 63 Nigula bog $20018 \delta^{\lambda} 15$ ㅇ leg. M. Smits det. C.-C. Coulianos. This record will be published in full by G. A. van Duinen (in prep.).

34 concinna See Coulianos (2003). Also recorded from LE 63 Nigula 3-8.x.1999 1ठ in light trap leg. M. Kruus.

39 lateralis Recorded as new to Estonia by Coulianos (1999) who, however, overlooked that it had been recorded by Ristkok (1994).

40 longipalis See Coulianos (2003).

49 reuteri See Coulianos (2003).

50 minutissima Järvekülg (2001) records "Plea minutissima Fabricius" from 68 localities in Estonian rivers. This certainly refers to Micronecta minutissima (L.). I have only seen Plea minutissima from ME 71 Kuldre 4.viii.1999 5 specimens leg. M. Marits. New to Estonia.

60 fucicola See Lindskog (1974).

65 palustris See Lindskog (1974). 
69 morio I have only seen this species from ME 98 Väägvere, Amme River 1.viii.1939 19 leg. L. Voore coll. IZB. The record from "Estonia" 1 q leg. G. Sumakov reported by Voore (1940) is Salda muelleri sec a specimen in coll. IZB.

70 muelleri Reported from Estonia by Flor (1860) (Salda littoralis part.) and Bianchi \& Kiritshenko (1923). I have seen only 1 ㅇ labelled "Estonia" leg. G. Sumakov, coll. IZB. No further locality is given.

71 sahlbergi Vilbaste (1955) recorded it from FL 32 Matsalu Nature Reserve (the islands of Liia and Väike-Härjamaa according to Rebassoo (1987)) 24.vi.1970. However, I have not found any specimens in coll. IZB.

77 aethiops First recorded from Estonia by Coulianos (2003), who mapped all known Estonian records.

82 distinguenda Recorded from Estonia (no localities given) by Kerzhner (1988).

87 epilobii New to Estonia. ME 87 Tiksoja bus stop 4.ix.2003, ME 87 The Dendropark at Estonian Agricultural University, Faculty of Forestry 4.x.2003 leg. C.-C. Coulianos. At both localities larvae and adults were very abundant on Epilobium hirsutum.

90 pilosus Recorded from ME 05 Rimmu 15.vii.1972 leg. I. M. Kerzhner (Lukashuk 1997).

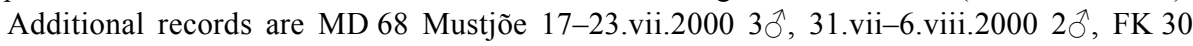

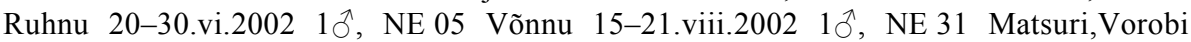
20-22.ix.2002 $1 \delta^{\lambda}$. All records in light trap and leg. et coll. A. Selin.

92 gothicus First recorded from LE 57 Pärnu 21.vii.1972 leg. I. M. Kerzhner (Lukashuk 1997). Also recorded from ME 65 Hurda 31.vii.2003 1ठ, NE 41 Korela 15.vii.1999 $2 \hat{\jmath}$ in light traps leg. et coll. M. Kruus.

93 morio First recorded from Estonia by Coulianos (2003), who mapped all known Estonian records.

97 trifasciatus See Coulianos (2003). Also recorded from LE 47 Audru 6-11.vi.2002 1ð in light trap leg. et coll. A. Selin.

102 reclairei Only recorded from ME 87 Tartu Botanical Garden 1998 (Coulianos 2003).

110 pilifer Recorded from EK 66 Viidumäe 8.vii.1984 1q leg. J. Vilbaste, EK 66 Viidumäe Nature Reserve 3.vii.1998 2ð 1 ㅇ on Molinia caerula leg. Coulianos. See Coulianos (2003).

124 adspersus First recorded from Estonia by Coulianos (1999). All specimens in earlier collections determined as Lygus gemellatus belong in fact to adspersus.

125 gemellatus Only recorded from EK 85 Saaremaa, Nasva. See Selin (2004).

127 punctatus In most collections this species has been mixed up with Lygus pratensis. First recorded by Coulianos (2003), who mapped all known Estonian records.

145 microphthalmus First recorded by Coulianos (1999). Also found in LF 63 Jalase 3.vii.1999 1 ㅇ leg. M. Marits.

148 tepastus First reported by Coulianos (2003), who mapped all known Estonian records.

152 binotatus First reported by Coulianos (2003), who mapped all known Estonian records.

159 erratica Reported from FL Matsalu Nature Reserve (Vilbaste 1985) and LF 30 Avaste mire (Vilbaste 1955) but I have seen no specimens from these localities in coll. IZB. The record from Ruhnu (Luig \& Talvi 1993) belongs to Notostira elongata sec specimens in coll. ZMT.

177 vittipennis First recorded from Estonia, Tartu leg. Morowitz by Lukashuk (1997). Additional records are from ME 65 Vellavere 1990, 1995, Tepripalu 1996 leg. M. Marits.

179 luridus See Coulianos (2003).

181 steganoides First recorded from Estonia by Coulianos (2003).

189 fulvicollis ME 87 Tähtvere 27.vii.1952 $2{ }^{\lambda} 2$ ㅇ leg. V. Maavara coll. IZB. New to Estonia.

191 sphegiformis The only record is from Saaremaa July $18519 \AA 5 q$ leg. Flor (Flor 1860).

192 erythrophthalmus Recorded from FL 41 Puise 28.vi.1970 1 ㅇ leg. J. Vilbaste (Vilbaste 1985).

204 viridinervis The only records are from FK 49 Puhtu 10.vii.1959 1ठ, 25.vii.1959 1ठ leg. S. Veroman, 16.vii.1960 1ठ leg. J. Vilbaste, coll. IZB. All specimens were found on Tilia cordata. 
212 mali Only recorded from ME 87 Tartu, Raadi 13.vii.1934 1§ึ on Malus domesticus leg. K. Leius coll. IPP.

229 viridiflavus Flor (1860: 631) reported under the name of Capsus setulosus a fifth instar larva of this species from Heiligensee (= ME 63 Pühajärve) but I have not found any specimen in coll. Flor in IZB. Additional records are MF 60 Kalevi, Kassinurme hills 6.viii.2000 10, Kalevi 9.ix.2002 1 ふै leg. K. Elberg, FK 59 Vatla, Lõo 2002 leg. A. Selin, ME 65 Hurda 2003, NE 41 Korela 1999, NE 00 Soodi 2003 leg. M. Kruus.

282 punctatus Reported from Tartu by Kerzhner (1981).

289 pilosus First recorded from Estonia by Coulianos (2003).

291 stigmatellus Recorded from NE 01 Võru by Lukashuk (1997).

301 pulchella See Voolma (1986).

303 formicetorum In Estonia according to Bianchi \& Kiritshenko (1923). I have seen no specimens in Estonian collections.

305 obscuripes See Selin (2004). In 2003 also found in SE Finland (Albrecht et al. 2003).

319 tipularius Published records from FK 39 Muhu, ME 63 Pühajärve (Flor 1860), MF 96 Koolma (Voore 1998). Additional records in IZB from ME 87 Tartu 1930 (G. Sumakov) and NE 04 Kiidjärve 6.viii.1949 (J. Vilbaste).

323 montivagus Reported by Vilbaste (1985) from FL 51 Matsalu Nature Reserve, 23.v.1961 leg. V. Ahas. In the card-index in IZB the following record is noted: FL 20 Muhu, Painase 5.vii.1961 leg. A. Vilbaste. I have seen no specimens in coll. IZB.

326 equestris In coll. IZB is 10 labelled "Dorp." (Dorpat = Tartu) det. G. Sumakov. This is an old and most probably accidental record as the principal food-plant of the species (Vincetoxicum hirundinaria) does not occur in this part of Estonia. On eastern Saaremaa on localities where this plant is abundant I have never found equestris. This is notable as the species is very common and abundant on the Baltic islands of Gotland and Öland and is known as a strong flier.

338 dispar See Coulianos (2003).

341 typhae See Coulianos (2003). Also found in ME 87 Tiksoja bus stop 4.ix.2003 2 + on Typha latifolia leg. C.-C. Coulianos.

346 costalis Reported from "Estonia" by Péricart (1998) according to specimens in coll. Zool. Mus. St. Petersburg. He does not report Camptotelus lineolatus from Estonia, a species recorded by Coulianos (1999).

363 rolandri See Selin (2004).

384 alboacuminatus The only record is from Dorpat (= ME 87 Tartu) reported by Flor (1860).

391 apterus See Elberg (2003). Also found in NE Võnnu 2002 leg. A. Selin, ME 65 Hurda 2003 leg. M. Kruus.

393 dahlmannii Only recorded from "Hapsal" (= FL 43 Haapsalu) by Jakovlev (1882).

394 laticornis See Selin (2004).

427 reticulata See Coulianos (2003). In 2001 and 2003 also found in SE Finland in sea-drift (Albrecht et al. 2003).

428 lineatum Elberg (1996) mapped the five records known up to 1989. Further records are EK 97 Saaremaa, Kaali 1997, FK 77 Tõstamaa park 2002 leg. K. Elberg, NE 15 Järvselja 2002 leg. A. Selin, NE 21 Piusa 2002 leg. G. Miländer.

430 macrocephalus Recorded from ME 86 Ihaste 3.viii.1990 391 larva leg. M. Marits (Coulianos 2003).

436 aeneus See Selin (2004). In 2003 also found in SE Finland in seadrifted material (Albrecht et al. 2003).

438 aliena Recorded from NE 17 Virvisaare 26.v.1949 1ठ leg. J. Vilbaste, ME 87 Tähtvere 16.vi.1951, LE 58 Nurme bog 25.v.1952 3 ^ leg. V. Maavara, LE 69 Suursoo 7.ix.1953 2q, LF 55 Leidissoo 10.ix.1953 1ð̊, MF 05 Muraka bog 17.vi.1953 1ठ all leg. J. Vilbaste. See Vilbaste (1955). 


\section{ACKNOWLEDGEMENTS}

I wish to express my gratitude to Dr. Kaupo Elberg (Tartu) and to Dr. Mati Martin (Institute of Zoology and Hydrobiology, University of Tartu) for their keen and never failing interest and help in my studies of the Estonian true bugs including good companionship on field studies and collecting trips and for letting me study their collections. Kaupo Elberg has also made valuable comments on a first version of this paper.

My best thanks also go to the following persons for information and for permitting me to study their private collections and museum collections in their care: Mikk Heidemaa (Institute of Plant Protection, Estonian Agricultural University, Tartu), Märt Kruus (earlier in Estonian Museum of Natural History, Tallinn, now Tartu), Jaan Luig (Zoological Museum, University of Tartu), Mati Marits (University of Tartu), Allan Selin (Tallinn), Hans Silfverberg (Zoological Museum, University of Helsinki), Tõnu and Tiina Talvi (Viidumäe Nature Reserve, Lümanda) for facilites during my visits on Saaremaa, Henn Timm (Võrtsjärv Limnological Station), Jaan Viidalepp (Institute of Zoology and Botany, Estonian Agricultural University, Tartu) who also was kind to translate the English abstract into Estonian, Kaljo Voolma (Faculty of Forestry, Estonian Agricultural University, Tartu), and Heino Õunap (Estonian Forest Research Institute, Tartu).

\section{REFERENCES}

Albrecht, A., Söderman, G., Rinne, V., Mattila, K., Mannerkoski, I., Karjalainen, S. \& Ahlroth, P. 2003. New and interesting finds of Hemiptera in Finland. Sahlbergia, 8, 64-78.

Aukema, B. \& Rieger, C. (eds.) 1998-2001. Catalogue of the Heteroptera of the Palaearctic Region. Vol. 1-4. Netherlands Entomological Society, Amsterdam.

Bianchi, V. I. \& Kiritshenko, A. N. 1923. Heteropteran Insects. Gos. Izd., Moskva \& Petrograd (in Russian).

Coulianos, C.-C. 1999. Some Hemiptera-Heteroptera new to Estonia. Proc. XXIV Nordic Congress Entomology, 203.

Coulianos, C.-C. 2003. Records of true bugs (Hemiptera-Heteroptera) new to Estonia with some biological notes. Sahlbergia, 9, 55-63.

Elberg, K. 1996. Putukad Eesti lõunapiiril. Eesti Loodus, 7, 262-264.

Elberg, K. 2003. Punalutikas laiendab levilat. Eesti Loodus, 6, 126.

Flor, G. 1860. Die Rhynchoten Livlands. Vol. 1. Schulz, Dorpat.

Flor, G. 1861. Die Rhynchoten Livlands. Vol. 2. Karow, Dorpat.

Haberman, H. 1933a. Uue nokalise leiust Eestis. Eesti Loodus, 1(1), 11-13.

Haberman, H. 1933b. Veel nokalisest Aphelocheirus montandoni Horv. Eesti Loodus, 1(1), 67.

Haberman, H. 1934a. Ahja jõe ülemjooksu põhjafaunast. Eesti Loodus, 2(3), 49-52.

Haberman, H. 1934b. Vellavere Külajärvest. Eesti Loodus, 2(5), 103-106.

Haberman, H. 1935. Keblaste tammik. Eesti Loodus, 3(5), 127-129.

Haberman, H. 1936a. Andmeid Pühajärve kalda- ja põhjafaunast. Eesti Loodusuurijate Seltsi aastaraamat, 42, 1-22.

Haberman, H. 1936b. Kahe huvitava veenokalise leide 1935. a. suvel. Eesti Loodus, 4(2), 74. 
Haberman, H. 1937a. Treppoja. Eesti Loodus, 5(1), 1-5.

Haberman, H. 1937b. Kostivere urgete loomastikust. Eesti Loodus, 5(5), 181-183.

Haberman, H. 1938. Selgrootud. In Läänemaa. Maateaduslik, majanduslik ja ajalooline kirjeldus (Haberman, H., Kant, E., Kruus, H., Luha, A. \& Tammekann, A., eds.), pp. 81-91. Eesti Kirjanduse Selts, Tartu.

Jakovlev, V. E. 1882. Contributions to the fauna of the Heteropterous insects of Russia and the neighbouring countries. Bull. Soc. Imp. Natural. Moscou, 57, 98-112.

Järvekülg, A. 2001. Jõgede põhjaloomastik. In Eesti jõed (Järvekülg, A., ed.), pp. 158-186. EPMÜ Zooloogia ja Botaanika Instituut, Tartu.

Jüris, A. 1940. Kõnnu raba selgrootute faunast kodumaa rabade fauna näitena. 4. In Eesti loodusteadlaste päev. Ettekannete kokkuvõtted, pp. 45-46.

Kauri, H. 1934. Selgrootud. In Eesti. Maadeteaduslik, majanduslik ja ajalooline kirjeldus. VI. Saaremaa, pp. 98-102. Eesti Kirjanduse Selts, Tartu.

Kerzhner, I. M. 1981. Bugs of the family Nabidae. In Fauna USSR Rhynchota, 13(2). Nauka, Leningrad (in Russian).

Kerzhner, I. M. 1988. New and Little Known Heteroptera from the Far East of the USSR (1987). Nauka, Vladivostok (in Russian).

Kiritshenko, A. N. 1951. True bugs of the European part of the USSR (Hemiptera). In Guides to the Fauna of the USSR, 42. Akad. Nauk SSSR, Moskva \& Leningrad (in Russian).

Leius, K. 1940. Kartulil esinevad nokalised ja nende tähtsus viirushaiguste ülekandmisel. Mitt. Versuchsstation für angew. Zoologie Univ. Tartu, 43, 1-4.

Lindskog, P. 1974. Distributional and systematic notes on Saldula fucicola (J. Sahlb.) and some other shore bugs of Eastern Fennoscandia (Heteroptera, Saldidae). Notul. Entomol., 54, 3356.

Luig, J. \& Talvi, T. 1993. Faunistilisi andmeid Ruhnu saare putukatest (Insecta). Eesti Looduseuurijate Seltsi aastaraamat, 73, 101-116.

Lukashuk, A. O. 1997. Annotated List of the Heteroptera of Belarus and Baltia. Russian Acad. Sci., Zool. Inst., St. Petersburg.

Maavara, V. 1957. Endla rabade entomofauna. Loodusuurijate Seltsi aastaraamat, 50, 119-140.

Mikkelsaar, N.-Ö. 1934. Naucoris cimicoides L. esmasleid Eestis. Eesti Loodus, 2(1), 16.

Mühlen, M. zur \& Schneider, G. 1920. Der See Wirzjerv in Livland. Arch. Naturkunde des Ostbaltikums, 14(1).

Mäemets, A. 1975. Kaitset vajavad haruldased liigid ja kooslused Eesti järvedes. In Eesti loodusharulduste kaitseks (Kumari, E., ed.), pp. 53-75. Valgus, Tallinn.

Pericart, J. 1998. Hémiptères Lygaeidae Euroméditerranéens. Vol. 2. Faune de France, 84 B. Paris.

Rebassoo, H.-E. 1987. Biocenoses on the Islets in the Eastern Part of the Baltic Sea. Akad. Nauk Estonskoj SSR (in Russian).

Remm, H. 1966. Putukate välimääraja I (Apterygota, Palaeoptera, Hemimetabola). Tartu Riiklik Ülikool, Tartu.

Ristkok, J. 1994. Emajõe veestiku vooluvetest leitud hüdrobiondid. Eesti Loodusuurijate Seltsi aastaraamat, 75, 97-147.

Rõigas, P. 1975. Hiidüraski levikust ja kahjustusest. Metsanduslikud Uurimused, XII, 280-293.

Selin, A. 2004. Uusi lutikalisi (Heteroptera) Eesti faunas. Lepinfo, 15, 57-58 (in Estonian).

Sepp, L. 1939. Ranatra linearis'e L. esmasleid Koiva jões. Eesti Loodus, 7, 190-191.

Stichel, W. 1927. Zur Kenntnis der estländischen Hochmoorfauna. Hemiptera-Heteroptera. Sitzungsber. Naturforsch. Ges. Dorpat, 33(2) (1926), 92-97.

Timm, H., Möls, T., Kangur, K. \& Timm, T. 1999. Littoral macroinvertebrates in some small lakes in Estonia. In Biodiversity in Benthic Ecology (Friberg, N. \& Carl, J. D., eds.), pp. 133-139. National Environmental Res. Inst. Technical Report No 266, Denmark.

Timm, T. 1965. Kagu-Eesti väikeste järvede põhjaloomastikust. Eesti Loodusuurijate Seltsi aastaraamat, 57, 83-102. 
Timm, T., Kangur, K., Timm, H. \& Timm, V. 1996. Macrobenthos of Lake Peipsi-Pihkva: taxonomical composition, abundance, biomass, and their relations to some ecological parameters. Hydrobiologia, 338, 139-154.

Timm, T., Kangur, K., Timm, H. \& Timm, V. 2001. Zoobenthos. In Lake Peipsi. Flora and Fauna (Pihu, E. \& Haberman, J., eds.), pp.82-99. Sulemees Publishers, Tartu.

Timm, V., Kangur, A. \& Timm, T. 1988. Saarema järvede põhjaloomastikust. Eesti Looduseuurijate Seltsi aastaraamat, 72, 95-103.

Valk, P. 1984. Liuskurlaste (Heteroptera, Gerroidea) bioloogiast ja levikust Eestis. Diploma thesis, Department of Zoology, University of Tartu.

Vilbaste, J. 1955. Eesti NSV soode rohurinde nokaliste faunast. Loodusuurijate Seltsi aastaraamat, 48, 104-121.

Vilbaste, J. 1958. Putukatest lumel. Eesti Loodus, 1, 21-25.

Vilbaste, J. 1970. Heteroptera. In Lääne-Eesti rannikualade loodus (Kumari, E., ed.), pp. 179-181. Valgus, Tallinn.

Vilbaste, J. 1973a. Heteroptera. In Võrtsjärv (Timm, T., ed.), p. 212. Valgus, Tallinn.

Vilbaste, J. 1973b. Revision of the collection of G. Flor. Eesti NSV TA Toim. Biol., 22, 15-28.

Vilbaste, J. 1979. The Hemipteroidea of the Vooremaa hardwood-spruce forest. Estonian IBP Report, $12,70-94$.

Vilbaste, J. 1985. Lutikalised (Heteroptera). In Matsalu rahvusvahelise tähtsusega märgala (Kumari, E., ed.), pp. 162-168. Valgus, Tallinn.

Voolma, K. 1986. Entomophages of European spruce beetle in Estonia. Metsanduslikud Uurimused, 21, 89-97 (in Russian).

Voolma, K. 1992. Kuuse käbikahjurid seemneaastatel. Mets ja Puu, 34(4), 11-15.

Voore, L. 1940. Andmeid rannalutikatest Eestis. Eesti Loodus, 8(2), 77-83.

Voore, V. 1998. Matkal Alutaguses. Eesti Looduseuurijate Seltsi aastaraamat, 78, 186-188.

\section{Eesti lutikaliste (Hemiptera-Heteroptera) annoteeritud nimestik levikuandmetega}

\section{Carl-Cedric Coulianos}

Viimaste aastate välitööde, teadusasutuste ja erakollektsioonide läbitöötluse ning faunistilise kirjanduse põhjal koostatud Eesti lutikaliste nimestikus on 454 liiki; nende levik on toodud tabelis $100 \times 100 \mathrm{~km}$ UTM-ruutude kaupa. On esitatud detailsemad kommentaarid 58 liigi leviku kohta. On lisatud kommenteeritud nimestik liikidest, mis on Eestist ekslikult publitseeritud. Järgmised liigid avaldatakse Eesti faunale uutena: Plea minutissima (Leach), Dicyphus epilobii Reuter ja Globiceps fulvicollis Jakovlev. 